University of Louisville

ThinkIR: The University of Louisville's Institutional Repository

Electronic Theses and Dissertations

$5-2021$

\title{
PDE4 inhibition: a novel therapeutic strategy in liver fibrosis.
}

\author{
Mohamed Elnagdy
}

University of Louisville

Follow this and additional works at: https://ir.library.louisville.edu/etd

Part of the Digestive System Diseases Commons, Medical Sciences Commons, Pathological

Conditions, Signs and Symptoms Commons, and the Pharmacy and Pharmaceutical Sciences Commons

\section{Recommended Citation}

Elnagdy, Mohamed, "PDE4 inhibition: a novel therapeutic strategy in liver fibrosis." (2021). Electronic Theses and Dissertations. Paper 3611.

https://doi.org/10.18297/etd/3611

This Master's Thesis is brought to you for free and open access by ThinkIR: The University of Louisville's Institutional Repository. It has been accepted for inclusion in Electronic Theses and Dissertations by an authorized administrator of ThinkIR: The University of Louisville's Institutional Repository. This title appears here courtesy of the author, who has retained all other copyrights. For more information, please contact thinkir@louisville.edu. 
PDE4 INHIBITION: A NOVEL THERAPEUTIC STRATEGY IN LIVER FIBROSIS

By

Mohamed Elnagdy

MBBCH, Cairo University, 2015

\author{
A Thesis \\ Submitted to the Faculty of the \\ School of Medicine of the University of Louisville \\ in Partial Fulfillment of the Requirements \\ for the degree of \\ Master of Science \\ in Pharmacology and Toxicology \\ Department of Pharmacology and Toxicology \\ University of Louisville \\ Louisville, Kentucky
}

May 2021 

PDE4 INHIBITION: A NOVEL THERAPEUTIC STRATEGY IN LIVER FIBROSIS

\author{
By \\ Mohamed Elnagdy \\ MBBCH, Cairo University, 2015
}

A Thesis Approved on:

April 5 2021

By the following Thesis Committee:

Leila Gobejishvili, PhD

Shirish Barve, $\mathrm{PhD}$

Craig McClain, MD

David Hein, PhD

Wenke Feng, PhD

Claudio Maldonado, $\mathrm{PhD}$ 


\section{DEDICATION}

I dedicate this thesis to my wife (Aya Eissa). You are a hero; thank you for always

being my supporter for my endless ambitions. To my kids, Ellen, Rose, and Mazen, you are the joy of my life. 


\section{ACKNOWLEDGMENTS}

I want to thank my Ph.D. mentor, Dr. Leila Gobejishvili, for her guidance in my graduate training. She taught me research from the zero point with extreme patience and passion. For these reasons and others, I am very grateful for her mentorship. 


\begin{abstract}
PDE4 INHIBITION: A NOVEL THERAPEUTIC STRATEGY IN LIVER FIBROSIS

Mohamed Elnagdy
\end{abstract}

$4 / 23 / 2021$

Background: Liver fibrosis is accumulation of extracellular matrix (ECM) proteins due to chronic liver injury. Chronic hepatic damage can occur due to multiple causes including, alcohol, non-alcoholic steatohepatitis and chronic viral hepatitis. Liver fibrosis is a critical problem worldwide due to the extremely high incidence of alcohol associated liver damage and chronic viral hepatitis. Moreover, there is no FDA approved therapy for liver fibrosis. Activation and transdifferentiation of quiecent hepatic stellate cells (HSCs) to myofibroblasts (MFBs) is the main event contributing to liver fibrosis. This process involves two phases, the initiation and the perpetuation phases. Hepatocyte injury and inflammatory response result in the initiation phase. During this phase, several profibrotic mediators (e.g.trasforming growth factor $\beta 1$ (TGF $\beta 1)$ ) initiate the activation of HSCs. Activated MFBs deposit extracellular matrix proteins (ECM), including collagen and fibronectin. In perpetuation phase, MFBs proliferate and acquire a contractile/motile phenotype.

Earlier work done by our group showed that spontaneous in culture HSC activation was accompanied by an increase in phosphodiesterase 4 (PDE4), a cAMP degrading enzyme. 
We also showed that PDE4 inhibition by Rolipram attenuated fibrogenic signaling in a bile duct ligation liver fibrosis rat model. More recent studies done by our group showed that PDE4 inhibition by Rolipram attenuates $\mathrm{CCl}_{4}$ induced liver fibrosis in $\mathrm{C} 57 \mathrm{Bl} / 6$ mice. Rolipram decreased collagen deposition as demonstrated by Sirius red staining and hydroxyproline assay. We also observed attenuation of markers of HSC activation ( $\alpha$ smooth muscle actin) and motility/contractility (pMLC and EDN1). Importantly, downstream cAMP effectors including, protein kinase A (PKA) and exchange protein activated by cAMP (EPAC) were shown to have significant antifibrotic effects. Hence, we hypothesized that PDE4 inhibition prevents development of liver fibrosis by attenuating TGFß1-induced activation of HSCs.

Methods: For in vivo studies, C57Bl6/J mice were subjected to a repeated $\mathrm{CCl}_{4}$ injections twice a week for the duration of 4 weeks. One group of mice received Rolipram twice a week, day after $\mathrm{CCl}_{4}$ administration. For in vitro studies, LX2 human HSC line was used. A group of cells was pretreated with Rolipram and then, recombinant human TGF $\beta 1$ was added. Cells were collected at early time points (30 and 90 minutes) and late timepoint (24 hours). RNA was extracted by trizol method from LX2 HSCs and, expression of several fibrotic genes was assessed using quintitative RT qPCR. We also made whole cell lysates from LX2 cells (late timepoint) using Radioimmunoprecipitation assay buffer (RIPA) buffer as well as nuclear and cytoplasmic lysates from early time points. Protein lysates were used in western blot analysis to assess expression of relevant proteins as well as early TGF $\beta 1$ signaling changes. We also performed scratch assay to examine the effect of Rolipram on HSC motility/contractility. In chronic $\mathrm{CCl}_{4}$ mouse model, We performed 
proteomic analysis to examine the affected proteins and pathways by PDE4 inhibition in liver fibrosis.

Statistical analysis was done using one-way ANOVA and unpaired t-test $\left({ }^{*} \mathrm{p}<0.05,{ }^{* *} \mathrm{p}\right.$ $<0.01$ and $\left.{ }^{* * *} \mathrm{p}<0.001\right)$.

Results: Proteomic analysis showed a significant effect of PDE4 inhibition on liver fibrosis pathways as well as pathways related to cytoskeleton remodeling, cell adhesion and motility/contractility. In LX2 cells, Rolipram attenuated TGFß1-induced HSC activation by decreasing phosphorylation of SMAD3 and HSC activation marker (aSMA). Rolipram also attenuated TGFß1-induced HSC motility/contractility by decreasing phosphorylated myosin light chain (pMLC) and endothelin-1 expression. Importantly, the effect of Rolipram on cell motility was validated by the results of the scratch assay which showed a significant decrease in percentage of wound closure at 24 hours.

Conclusions: These results demonstrate that the PDE4 inhibition attenuates the initiation and perpetuation of liver fibrosis thorugh reduction of TGF $\beta 1$-mediated HSC activation and motility/contractility 
TABLE OF CONTENTS

ACKNOWLEDGMENTS ..................................................................................... iv

ABSTRACT …

LIST OF FIGURES .........................................................................................

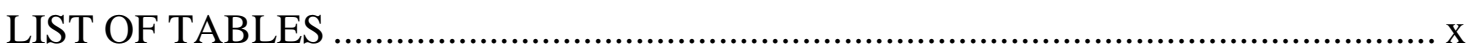

CHAPTER 1: INTRODUCTION AND BACKGROUND …………………............. 53

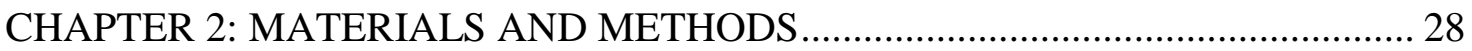

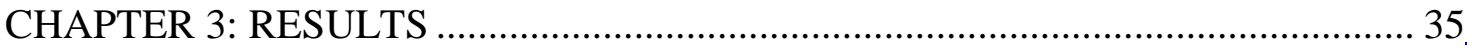

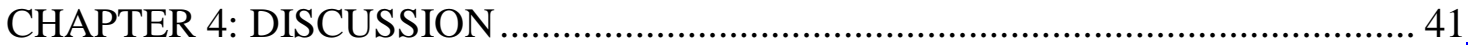

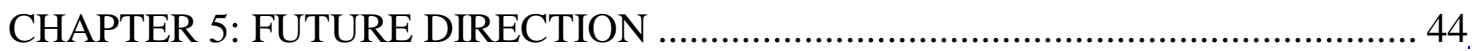

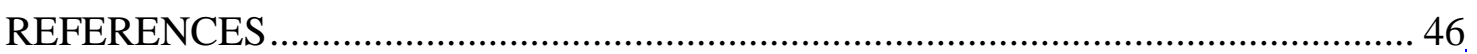

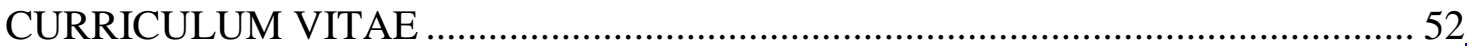




\section{LIST OF FIGURES}

FIGURE 1. spectrum of liver fibrosis. Nature reviews, immunology

FIGURE 2. Hepatic stellate cell activation and liver fibrosis

FIGURE 3. HSCs activation involves the initiation and perpetuation process

FIGURE 4. TGF $\beta 1$ canonical signaling pathway

FIGURE 5. cAMP signaling pathway

FIGURE 6. $\mathrm{CCl}_{4}$ experimental mice model/experimental design

FIGURE 7. PDE4 inhibition attenuates TGF $\beta 1$ induced LX2 HSC activation

FIGURE 8. PDE4 inhibition attenuates TGF $\beta 1$-induced HSC motility/contractility through decreasing pMLC and EDN1 expression

FIGURE 9. PDE4 inhibition significantly decreased wound healing capacity in LX2 HSCs treated with TGF $\beta 1$

FIGURE 10. Summary figure 


\section{LIST OF TABLES}

TABLE 1. qPCR primer sequences

TABLE 2. PDE4 inhibition significantly affects pathways related to liver fibrosis 


\section{CHAPTER 1}

\section{INTRODUCTION AND BACKGROUND}

\subsection{Liver histology and functional anatomy:}

As reviewed in [1], hepatic parenchyma is composed of multiple cells, including hepatocytes and endothelial cells as well as non-parenchymal cells, including, hepatic stellate cells (HSCs) and Kupffer cells (KCs). Hepatocytes are arranged in cords around central veins radiating towards a peripheral hexagonal stromal frame forming the classic hepatic lobule. At the corners of this lobule exist the portal triad, including; portal vein, bile ductule, and hepatic arteriole. Blood and bile flow in opposite directions in the hepatic lobule. The bile, secreted by hepatocytes in bile canaliculi, flows peripherally towards the bile ductule. The blood entering the hepatic artery and portal vein flows into the liver sinusoid toward the central vein. The sinusoid is the liver's microvascular unit composed of fenestrated endothelial cell lining separated from hepatocytes by space of Disse. This space harbors the hepatic stellate cells which exist in a quiescent phenotype. Quiescent HSCs maintain a low-density extracellular matrix for proper exchange of nutrients/metabolites between hepatocytes and the bloodstream. 
As reviewed in [1, 2], hepatocytes have multiple crucial functions to maintain normal body homeostasis and physiology. Their functions include protein synthesis and storage, metabolic functions, synthetic functions, and detoxification functions. Hepatocytes synthesize plasma proteins, e.g., albumin and globulins, as well as fibrinogen, prothrombin, and other coagulation factors. They also synthesize lipoproteins for lipid metabolism, ceruloplasmin for copper transport, transferrin for iron transport, and complement factors that have an important immune function. They are also axial metabolic cells involved in carbohydrate metabolism through glycogenesis, glycogenolysis, and gluconeogenesis. They synthesize chylomicrons from fats absorbed from the intestine and handle serum low density lipoproteins (LDL) and very low density lipoproteins (VLDL), and protein metabolism by detoxifying ammonia generated from amino acid metabolism into urea through the urea cycle. Hepatocytes also synthesize bile, which is involved in the emulsification of fats, and this is an essential step for fat absorption. Hepatocytes also can metabolize and detoxify xenobiotics as well as drugs and toxins/toxicants, e.g., insecticides.

Hepatic stellate cells are the resident mesenchymal cells of the liver. Activation of HSCs into contractile myofibroblasts (MFs) generates scar tissue causing fibrosis [3]. Apart from hepatic wound healing by liver fibrosis, HSCs have many other functions, including, storage of vitamin A as retinol granules, immunoregulation, and regulation of portal blood flow [3].

Kupffer cells (KCs) are the resident hepatic macrophages derived from circulating blood monocytes and are part of the innate immune response [4]. KCs are mainly responsible 
for the initial response to any hepatic antigen, a microorganism, or a toxin [4]. They have a phagocytic function by which they can engulf and process the antigen, then presenting its degradation products to $\mathrm{T}$ helper cells for activation of a specific immune responses against the antigen. KCs also secrete cytokines through which they interact with other immune cells and HSCs, initiating their activations [4].

The hepatic artery and portal vein are responsible for the blood supply of the liver. The blood enters from the portal triad and moves towards the central vein through hepatic sinusoids to allow the exchange of substances between hepatocytes and the bloodstream through the endothelial cells and space of Disse. Central veins drain in hepatic vein, which drains into the systemic venous circulation in the Inferior Vena Cava (IVC) [5].

\subsection{Introduction to Liver Fibrosis:}

As reviewed in [6], hepatic fibrosis is a wound healing process characterized by the accumulation of extracellular matrix (ECM) due to liver injury. In case of acute or selflimited injury, transient changes occur with hepatic regeneration and typical hepatic architecture restoration. However, if the damage is sustained, fibrosis and scar formation outweigh the hepatic regeneration, causing accumulation of scar tissue and fibrosis. This results in deterioration of hepatic function and end-stage liver cirrhosis, and can cause liver failure or hepatocellular carcinoma. Both liver cirrhosis and hepatocellular carcinoma have a poor outcome and high mortality rate. Progression to end-stage liver disease can take 5-50 years, depending on multiple factors, mainly genetic and environmental. 


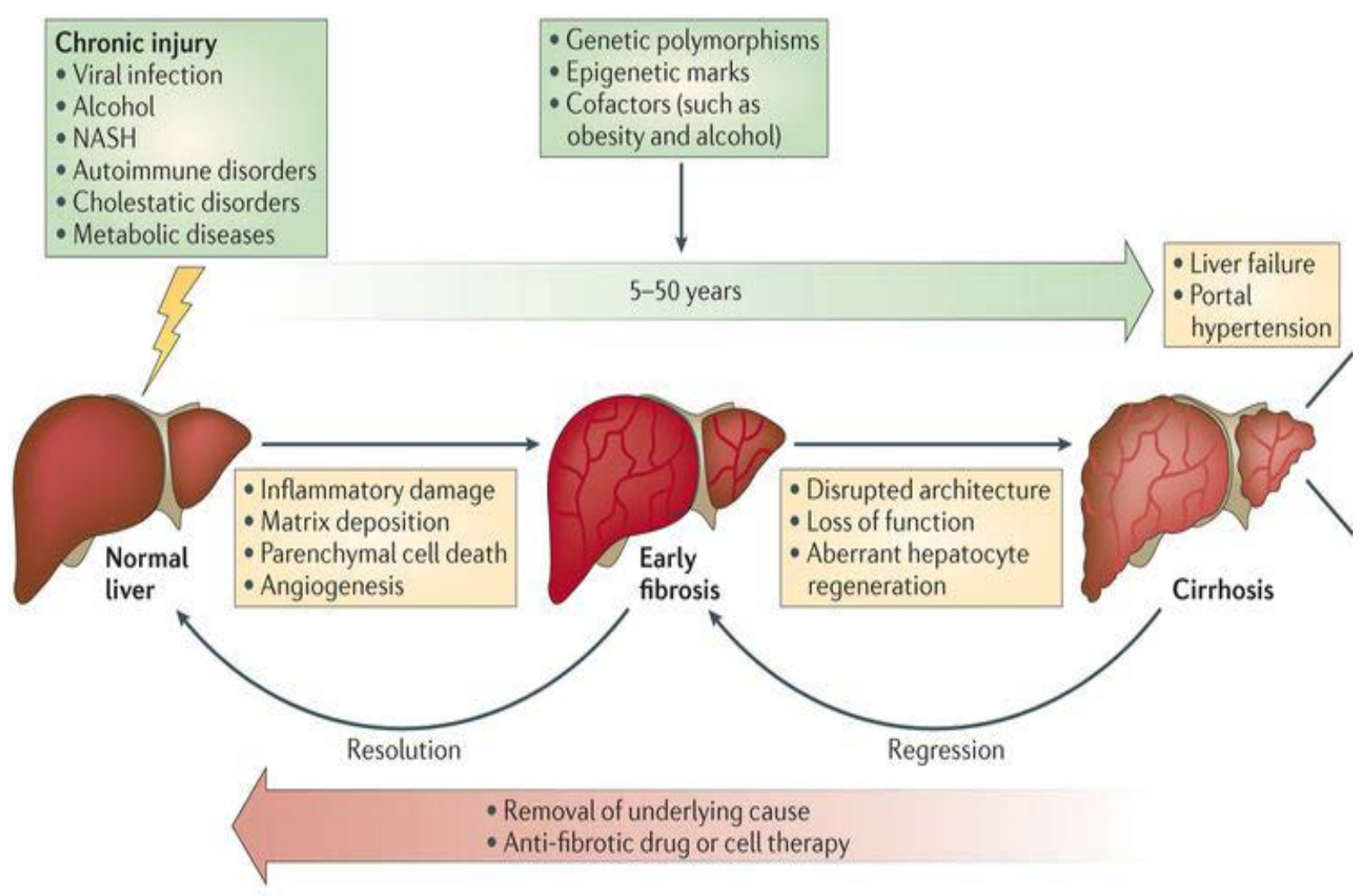

Figure 1. spectrum of liver fibrosis.

Adopted from: Pellicoro, A., Ramachandran, P., Iredale, J. et al. Liver fibrosis and repair: immune regulation of wound healing in a solid organ. Nat Rev Immunol 14, 181-194 (2014). https://doi.org/10.1038/nri3623

According to the 2015 national survey on drug use and health, $70.1 \%$ of adults aging 18 years or older reported that they consumed alcohol regularly in the past year. An estimated 88,000 people die from alcohol-related liver diseases annually [7]. An estimated 2.7-3.9 million people in the USA have hepatitis C virus (HCV) infection, with rates even higher in other countries, e.g., Egypt [8]. The prevalence of chronic viral hepatitis due to HCV and HBV (hepatitis B virus) is more than 5 million in the USA. Around $75 \%$ of HCV patients are unaware of their infection. Chronic viral hepatitis had a 
mortality rate of around five deaths/100,000 in 2014 [9]. Non-alcoholic fatty liver disease occurs in approximately $30 \%$ of the US population, about 100 million individuals in the US [10]. All the previously mentioned liver diseases are considered causes of chronic liver injury, and the usual cause of mortality is liver cirrhosis/liver cell failure on top of liver fibrosis. Thus, studying liver fibrosis and finding new drug targets to attenuate its progression is extremely important because, as of now, there is no FDA-approved therapy for liver fibrosis.

\subsection{Clinical and pathological features of liver fibrosis:}

Liver fibrosis occurs due to ongoing chronic hepatic injury. Many causes lead to liver fibrosis, including: alcoholic liver injury, which is the most common cause in the US; chronic viral hepatitis (HCV and HBV); obesity-induced liver injury; and toxicantinduced liver injury. Multiple pathological changes happen due to dysregulated liver fibrosis, which leads to the development of liver cirrhosis [6]. Deposition of ECM starts in the space of Disse, causing a loss of normal fenestrations existing in the hepatic sinusoids, in turn causing impairment of the typical metabolic exchange between the blood and hepatocytes in a pathological process called capillarization of the sinusoids [11]. Many pathological patterns have been described in liver fibrosis, including: 1) bridging fibrosis, which usually happens in chronic viral hepatitis; 2) perisinusoidal fibrosis associated with alcoholic and non-alcoholic liver injury; 3) biliary fibrosis or periductular fibrosis, which is caused by biliary tract diseases; and 4) centrilobular fibrosis which is caused mainly by conditions altering hepatic venous return to the systemic venous circulation [12]. 
As reviewed in [13], liver cirrhosis occurs due to an imbalance between liver injury/fibrosis and hepatocyte regeneration. Liver cirrhosis is the most common nonneoplastic cause of mortality among digestive diseases. Cirrhosis has two stages, starting with a compensated phase followed by a decompensated phase. This process leads to portal hypertension and liver cell failure, which can have many clinical manifestations including jaundice, esophageal varices, rectal hemorrhoids, spider nevi, and flapping tremors. Liver cell failure can end in hepatic coma and death due to the accumulation of ammonia in the blood, which is toxic to the CNS.

\subsection{Hepatic extracellular matrix: physiology and pathology}

In normal liver, extracellular matrix (ECM) shows a high dynamic regulation between formation and degradation in a process called ECM remodeling. ECM is essential to provide structural and functional integrity to hepatic parenchyma [14]. Typically, ECM constitutes about $3 \%$ or less of the liver tissue and $0.5 \%$ of liver weight. ECM includes collagen, proteoglycans, laminin, fibronectin, and matricellular proteins [15]. It forms the liver tissue framework, including, Glisson's capsule surrounding the liver, portal tracts, central veins, and space of Disse. In the space of Disse, a low-density basement membrane-like matrix is composed mainly of collagen types IV and VI. ECM also contains matrix metalloproteinases (MMPs) and tissue inhibitors of metalloproteinases (TIMPs) which are responsible for ECM remodeling [16].

During chronic hepatic injury, an imbalance occurs between ECM formation and degradation, causing thickening of stromal septae with changes in collagen types associated with collagen cross-linking [17]. In the space of Disse, disruption of 
physiological low-density matrix occurs with the replacement of collagen type IV and VI with fibrillary collagen types, such as collagen type I and type III, and fibronectin. This leads to alteration of sinusoidal lumen physiology and hepatic function [18]. ECM can also modulate HSCs activation and proliferation and promote their growth, activation, and migration. Thus, ECM's role in liver fibrosis is believed to be bi-directional. For example, integrins are transmembrane receptors with a head domain that binds ECM components and cell adhesion molecules [19]. Integrins were shown to modulate and interfere with transforming growth factor $\beta$ (TGF $\beta$ ) and platelet-derived grown factor (PDGF) signaling pathways involved in liver fibrosis in which different integrin families were shown to be upregulated [20,21]. HSCs and endothelial cells were shown to express two critical molecules from the ADAM family of integrins, ADAMSTS-13 and ADAMSTS-1 [22]. Another important integrin molecule is the Discoidin domain receptor 2 (DDR2), activated primarily by collagen types I, II, III, and V, contributing to HSC activation and epithelial to mesenchymal transition [23].

\subsection{Liver fibrosis and hepatic stellate cells}

Hepatic stellate cells (HSCs) were identified as the primary fibrogenic cells in the liver. Advances in the clarification of HSC biology is the main bridge towards effective antifibrotic therapy in the near future.

The liver is a regenerative organ; however, sustained parenchymal injury leads to the activation of wound healing fibrotic process and deposition of extracellular matrix (ECM) proteins [6]. Activated HSCs are the main fibrogenic cell population in the liver tissue [6]. HSC activation leads to excessive deposition of fibrillary collagen type I and 
III, showing a 3-10 fold increase in fibrotic liver tissue [18]. Besides collagen, other ECM proteins, e.g., Fibrillin, Fibronectin, and sulfated proteoglycans, are also deposited in liver fibrosis [18]. HSCs reside in the sub-endothelial space of Disse between the cords of hepatocytes and liver sinusoidal endothelial cells, one of the main ECM deposition sites during liver fibrosis [3].

The primary site of injury, which varies according to liver injury, is another site of ECM protein-deposition. This leads to the different patterns of fibrosis which were described before.

There are multiple expected pathological outcomes associated with liver fibrosis. ECM deposition in the space of Disse impairs the process of solute exchange between the hepatocytes and the plasma [11]. Loss of fenestrae in the liver sinusoids in a process known as capillarization is the main factor responsible for impaired solute exchange [11]. The course of most chronic liver diseases takes decades until advanced fibrosis develops. During this course, the patient is usually asymptomatic or has minimal symptoms making it hard for early detection [24]. Advanced fibrosis also leads to liver failure, portal hypertension, and increased hepatocellular carcinoma risk on top of liver cirrhosis [25].

\subsubsection{Cell biology of liver fibrosis}

The contractile and highly secretory hepatic myofibroblasts (MFBs) are considered the primary cells responsible for liver fibrosis [26]. MFBs transdifferentiate from quiescent resident primary HSCs as well as from periportal fibroblasts [27]. Quiescent HSCs represent $5-8 \%$ of cells in the healthy liver contributing to hepatic development, regeneration, immune responses, angiogenesis, and vitamin A storage [3]. HSCs can be 
differentiated from portal fibroblasts morphologically by vitamin A droplets. They can also be differentiated genetically by expressing desmin, glial fibrillary acidic protein, L-rat, Hand-2, Vimentin, PDGFR- $\beta$, cytoglobin, and Reelin [28, 29]. They also differ based on the type of ECM protein each produces. HSCs derived MFBs deposit fibrillin positive elastin negative ECM while activated portal fibroblasts deposit fibrillin positive elastin positive ECM [30].

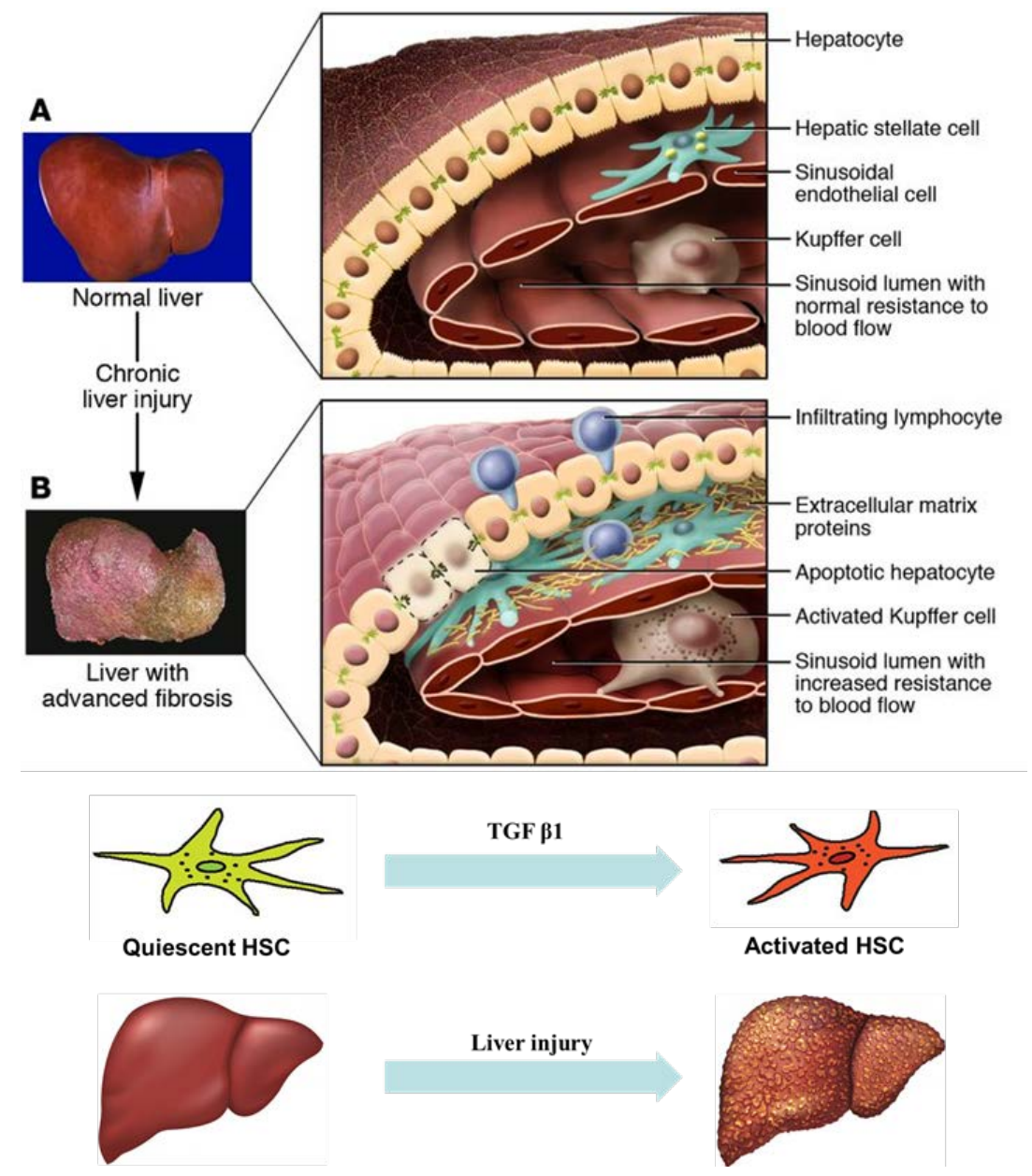

Figure 2. Hepatic stellate cell activation and liver fibrosis

Adopted from: Bataller R, Brenner DA. Liver fibrosis. J Clin Invest. 2005 
HSCs activation involves two phases, the initiation phase and the perpetuation phase [26]. In the initiation phase, upregulation of growth factor receptors and their signaling makes the quiescent HSCs more responsive to triggers and injury stimuli. This leads to the activation of HSCs in the zones of severe liver injury and inflammation [26]. Perpetuation is the process of amplification of the activated HSCs (MFBs) phenotype. This includes multiple distinctive features, such as proliferation, contractility, fibrogenesis, and matrix deposition [26]. Apoptosis, senescence, or reversion to quiescence may follow perpetuation if the underlying injury is resolved early [31]. However, with the persistence of injury, an imbalance occurs between the hepatocyte regeneration and healing by fibrosis leading to the development of the pathological fibrosis associated with deterioration of liver function [32]. 


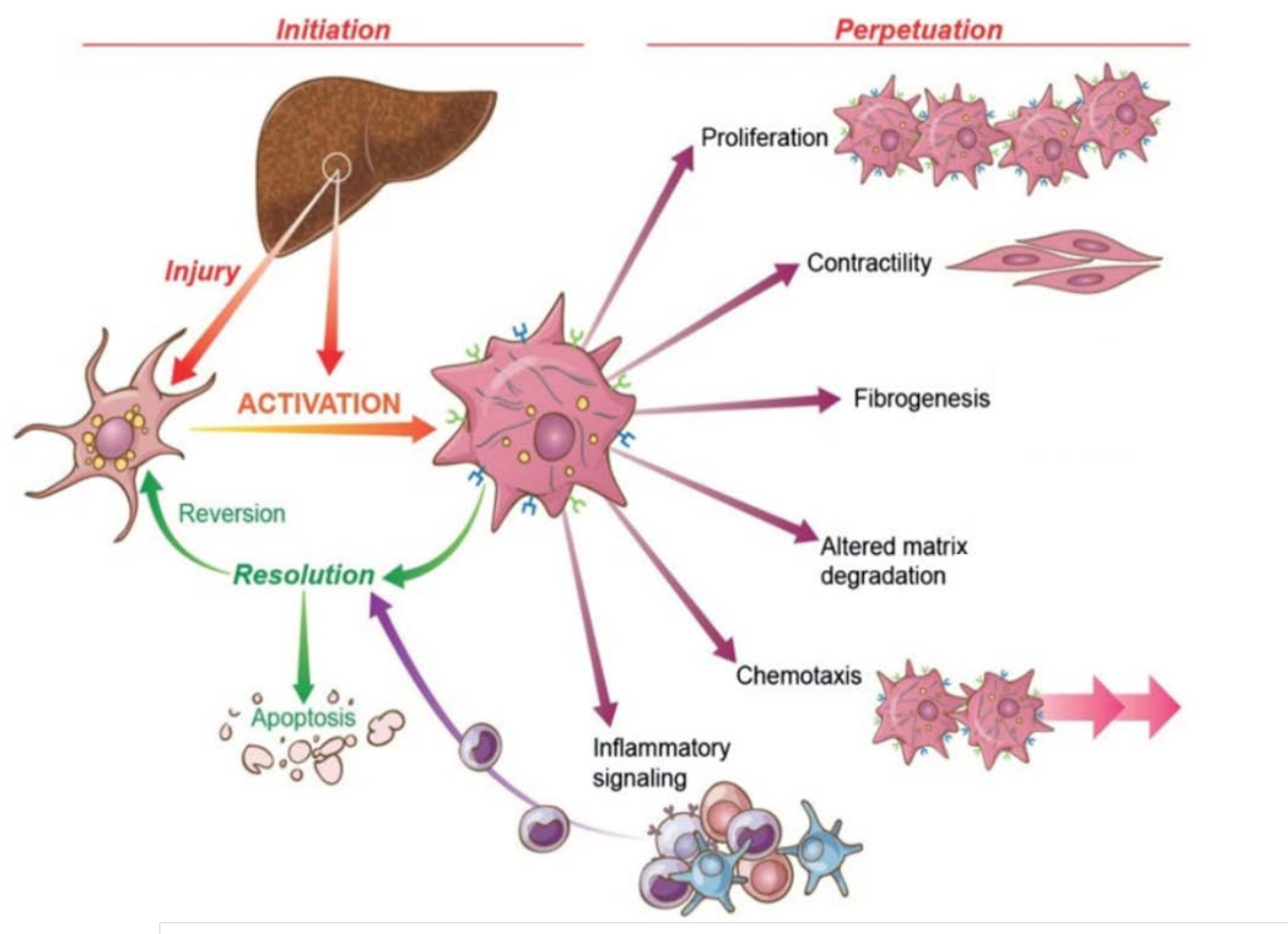

Figure.3 HSCs activation involves the initiation and perpetuation process that causes HSCs proliferation, contractility, fibrogenesis, and inflammatory signaling. Adopted from: Trivedi P, Wang S, Friedman SL. The Power of Plasticity-Metabolic Regulation of Hepatic Stellate Cells. Cell Metab. 2021 Feb 2;33(2):242-257. doi: 10.1016/j.cmet.2020.10.026. Epub 2020 Nov 23. PMID: 33232666.

Multiple biological factors play a role in the process of HSCs activation, including; Transforming growth factor $\beta$ (TGF $\beta$ ), Platelet-derived growth factor B (PDGF-B), Connective tissue growth factor (CTGF), Vascular endothelial growth factor (VEGF), Endothelin-1 (ET-1), Tissue inhibitors of metalloprotease (TIMP) and Matrix metalloproteases (MMPs). 
TGF $\beta$, mainly TGF $\beta 1$, is a potent regulator of HSCs proliferation, differentiation, and fibrogenesis. TGF $\beta 1$ is a homodimer that binds to TGF $\beta 1$ receptor forming heterotetrameric complexes. Transmembrane receptor serine/threonine kinase propagates the signal to downstream intracellular signaling molecules known as SMAD proteins. TGFß1 receptor phosphorylates SMAD2 \& SMAD3 proteins, which bind together with SMAD4 to form the SMAD complex, which translocates to the nucleus to mediate most of the TGF $\beta 1$ target effects. The significant impacts of TGF $\beta 1$ include 1) upregulation of expression of collagen I, II, and IV, fibronectin, and laminin, and 2) downregulation of collagenase protease inhibitors such as TIMP1, collagenase, and stromelysin [18, 33]. Aside from the induction of collagen synthesis through HSCs activation, TGF $\beta 1$ also increase HSCs motility/contractility [34]. TGFß1 was shown to increase phosphorylated myosin light chain (pMLC) by induction of myosin light chain kinase and inhibition of myosin light chain phosphatase. Phosphorylated myosin light chain protein is an activated form which couples with actin filaments and other focal adhesion proteins mediating activated HSC motility. TGF $\beta$ also induces mitogen-activated proteinase (MAPK) pathways (ErK, p38 MAPK, and JNK) independently of SMADs [35, 36]. Since systemic inhibition of TGF $\beta$ promotes carcinogenesis, liver or cell type-specific inhibition of TGF $\beta$ would be an ideal strategy for liver fibrosis treatment [37, 38]. 


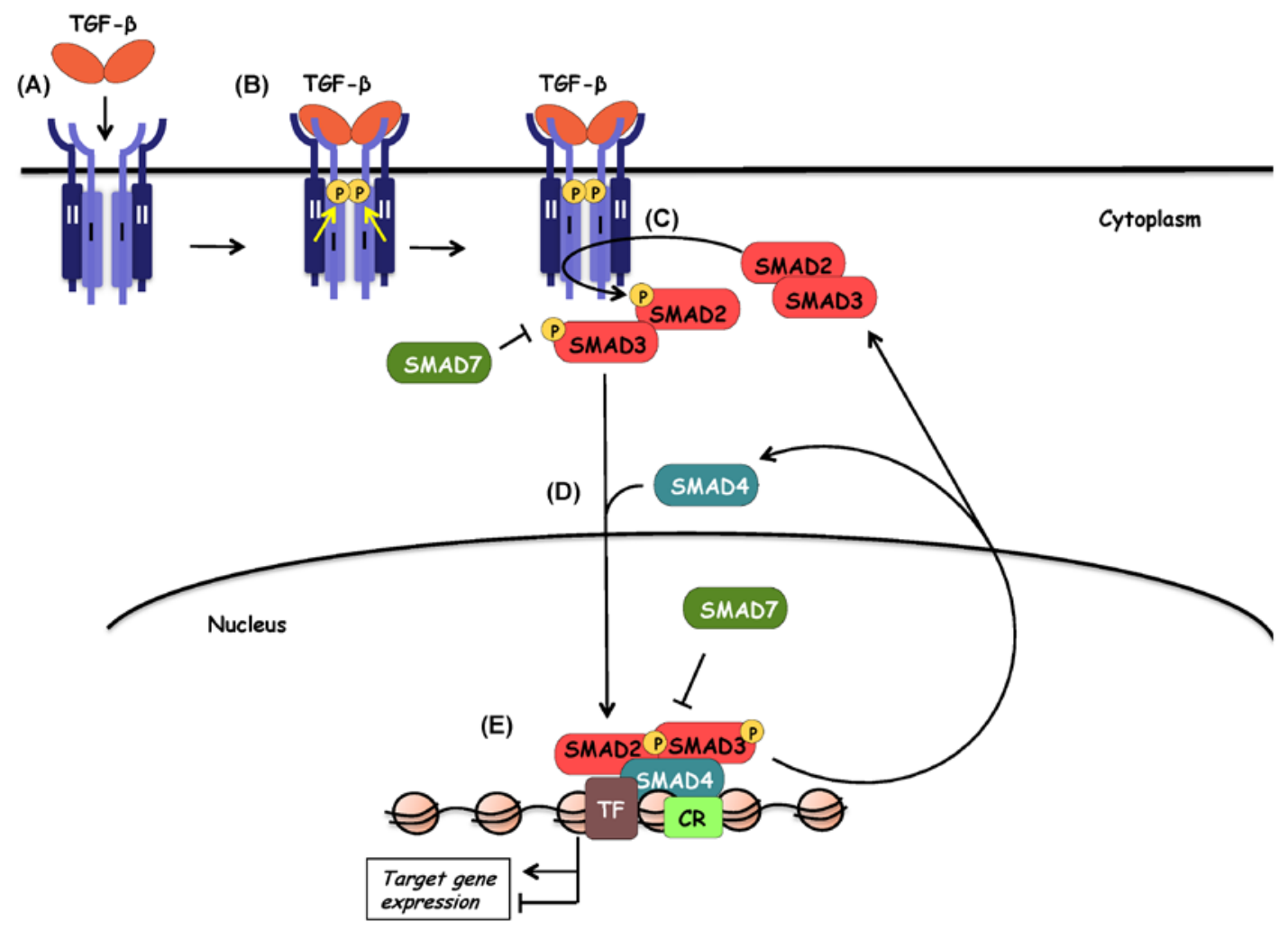

Figure 4. TGFß1 canonical signaling pathway.

Adopted from: Tzavlaki, K., and A. Moustakas, TGF- $\beta$ Signaling. 2020. 10(3): p. 487.

PDGF-B is the most potent mitogen and chemoattractant for HSCs. PDGF acts on PDGF receptor $\beta$, which is a tyrosine kinase receptor. Quiescent HSCs express $\alpha$ PDGF receptor with increased $\beta$ PDGF receptor expression after $\operatorname{HSCs}$ stimulation by TGF $\beta 1[39,40]$. PDGF receptor $\beta$ is upregulated during the initiation process, and this was shown by studies in HSCs in humans and rodents [39, 40], thereby amplifying PDGF-B signaling in HSCs. PDGF-B has multiple sources, including HSCs themselves, macrophages, and platelets [41]. In murine liver fibrosis models, knocking out PDGF receptor $\beta$ on HSCs led to attenuation of liver injury and fibrosis [42]. PDGF expression was also shown to be significantly upregulated after $\mathrm{CCl}_{4}$ administration to rats [43]. 
Endothelins (ETs) are a group of peptides formed by proteolytic cleavage of precursor propeptides by endothelin converting enzymes. ETs act on G protein-coupled endothelin receptors, causing many effects that play a significant role in liver fibrosis [44]. ET1 is a significant regulator of HSC contractility. It also acts as a potent vasoconstrictor, causing increased portal resistance associated with liver fibrosis [45]. It also plays a role in in vitro HSCs activation [46]. Studies also showed that endothelins might take part in collagen bands' contraction, leading to liver cirrhosis [45].

CTGF is an emerging target for antifibrotic drugs. CTGF is a profibrogenic protein expressed by HSCs in the injured liver, promoting fibrogenesis, adhesion, migration, and cell survival [47]. Inhibition of CTGF by human anti-CTGF antibodies are in clinical trials for pulmonary fibrosis (ClinicalTrials.gov ID \#NCT01217632).

Tissue inhibitors of metalloproteinases (TIMPs) TIMP1 and TIMP2 are produced by activated HSCs. TIMP1 has an antiapoptotic effect on HSCs mainly through induction of Bcl-2 antiapoptotic pathway, thus promoting cell survival [48]. HSCs also produce MMP2 and MMP9, and they disrupt the normal hepatic matrix to be replaced with fibrotic matrix $[49,50]$.

VEGF is mainly released from liver sinusoidal endothelial cells and HSCs in the injured liver. It induces HSCs proliferation, migration, and collagen production. It also mediates angiogenesis, which is a pathogenic process in advanced liver disease. It may also be a requirement for liver regeneration [51, 52]. 


\subsection{Cyclic AMP signaling}

As reviewed in [53], cAMP was the first second messenger to be identified and described in 1958 [54]. It is generated from ATP by adenylyl cyclase (AC) in response to various signaling molecules. There are nine transmembrane adenylyl cyclases (tmAC). They are differentially expressed and regulated to generate cell and stimulus-specific responses [55]. Transmembrane ACs are activated upon engagement to G protein-coupled receptors (GPCRs). In 1975, soluble AC was first described in the cytosol of rat testis and was later found in the nucleus, mitochondria, and centrioles. Soluble AC activity is regulated by intracellular levels of bicarbonate, calcium, and ATP [56].

Binding of GPCRs to their specific agonists leads to a conformational change. This change activates GPCR-bound heterotrimeric $\alpha \beta \gamma$ G protein, where GTP replaces GDP bound to the alpha subunit. The GTP-bound $\alpha$ subunit dissociates from the $\beta \gamma$ dimer. ACs are stimulated mainly by Gas dissociated subunit; however, some ACs are stimulated by the $\beta \gamma$ complex [57]. Generated cAMP can activate many effector molecules, including protein kinase A (PKA), guanine nucleotide exchange factor activated by cAMP (EPAC), and cyclic nucleotide-gated ion channels. PKA, the most extensively studied effector, is a complex of two regulatory (R) and two catalytic subunits (C). The binding of cAMP to two R subunits causes the $\mathrm{C}$ subunits to dissociate [58]. PKA acts on many cytosolic and nuclear substrates. PKA-mediated phosphorylation regulates the activity of numerous metabolic enzymes (e.g., glycogen synthase and phospholipase $\beta 2$ ). Regulation of gene expression by PKA is achieved by phosphorylation of cAMP response element binding protein (CREB), cAMP-responsive modulator (CREM), and activating transcription 
factor 1 (ATF1). Once phosphorylated, CREB binds to other cofactors, CREB binding protein (CBP) and p300, before binding to cAMP response elements on DNA. The CREM gene acts as a feedback inhibitor for inducible cAMP early repressor protein (ICER) [59, 60].

Another critical effector for cAMP is EPAC, with two genes (EPAC1 and EPAC2), and three transcript variants for each gene [61]. EPAC, as a cAMP sensor, was discovered 30 years after the discovery of PKA, in 1998. EPAC2 is mainly expressed in the liver, brain, pancreas, and adrenal gland, while EPAC1 is expressed ubiquitously. Binding of cAMP to EPAC leads to activation of the Ras GTPases (Rap1 and Rap2), known as cAMPregulated guanine exchange factors. In addition to their differential cellular expression, subcellular localization of both EPAC1 and EPAC2 determines the specificity of cAMP signaling (reviewed in [61]). They serve as interacting partners for multiple proteins and regulate numerous functions in various organs and systems, including the digestive and immune systems [61].

cAMP signaling is fine-tuned by a specific group of enzymes known as phosphodiesterases (PDEs) [62-64]. PDEs are a large family of ubiquitously expressed enzymes responsible for the termination of cAMP signaling through catalyzing cAMP hydrolysis reaction to AMP. There are 11 different PDE families (PDE1 to PDE11), and they differ in their tissue distribution, substrate specificity, subcellular localization, and catalytic properties [62, 64, 65]. They can be grouped according to their substrate specificity: cAMP-specific PDEs, including PDE4, PDE7, and PDE8; cGMP-specific PDEs, including PDE5, PDE6, and PDE9; and dual-specificity PDEs including PDE1, 
PDE2, PDE3, PDE10, and PDE11. Cells might express several PDE isoforms in various subcellular locations; however, some cells show relatively abundant expression of specific PDEs (e.g., PDE6 in the retina). Moreover, expression changes and mutations of multiple PDE enzymes have been linked to several disease states [66].

It is essential to point out that the generation of cAMP and downstream signaling is specific to the stimulus and cell type. This specificity is ensured by the presence of cellspecific GPCRs coupled with Gs proteins and AC. Some ACs reside in lipid rafts, while others are in various cellular compartments [67]. Additionally, A-kinase anchoring proteins (AKAPs) can interact with ACs to regulate cAMP signaling by creating a scaffold with PKA and its target [67, 68]. Notably, the fine-tuned and specific cAMP signaling is achieved by the co-existence of AC with a particular PDE isoform in a scaffold (compartmentalized cAMP signaling). The scaffold ensures PDE spatial, temporal, and compartmental downstream signaling activation. It is becoming increasingly evident that cAMP signaling uses cellular compartmentalization to coordinate the cellular functions under its control. This compartmentalization of cAMP was first recognized in the early 1980s in studies on cardiac myocytes. In these studies, researchers showed that cAMP levels increased in response to PGE1 and isoproterenol. However, only isoproterenol increased contractility [69]. These studies led to the hypothesis that the cAMP signaling pathway is organized in specific intracellular compartments to regulate its downstream targets and physiological outcomes. Studies using fluorescence resonance energy transfer (FRET) to visualize cAMP microdomains in a living cell later proved support for this hypothesis [70-72]. A large family of cAMPspecific PDEs provides additional specificity of cAMP signaling. Several studies using 
PDE4A, B, and D knockout mice have shown that these enzymes have non-redundant roles in various cellular and tissue responses [62, 73-76]. Interistingly, it has also been demonstrated that PDE4B plays an essential role in endotoxin-induced TNF production and toxicity, while PDE4A and D have no effect $[74,76]$

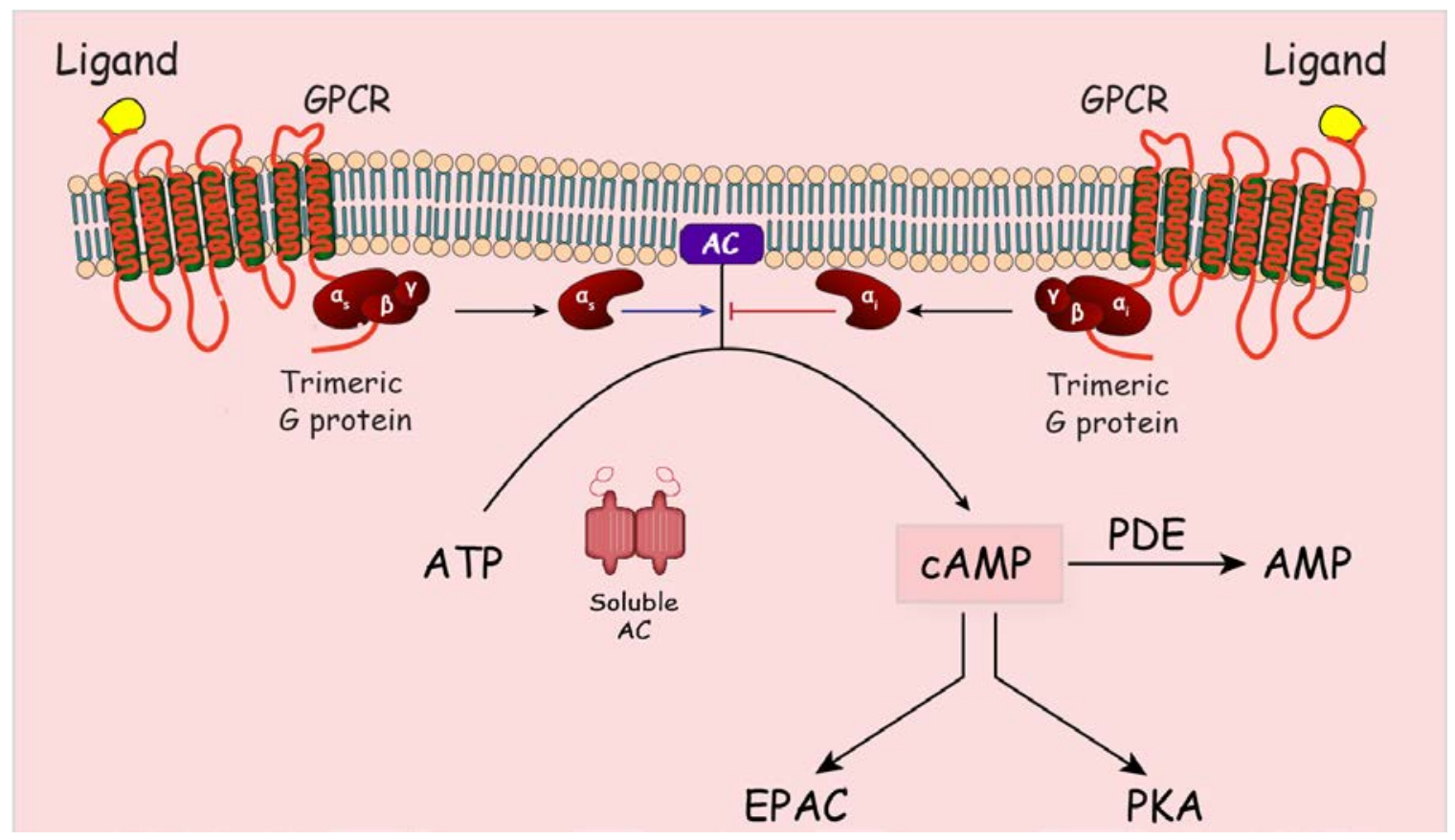

Figure 5. Schematic diagram of cAMP signaling pathway.

Elnagdy M, Barve S, McClain C, Gobejishvili L. cAMP Signaling in Pathobiology of Alcohol Associated Liver Disease. Biomolecules. 2020 Oct 11;10(10):1433. doi:

10.3390/biom10101433. PMID: 33050657

\subsection{Cyclic AMP and Liver fibrosis}

As reviewed in [53], The antifibrotic effects of cAMP effector molecules PKA and EPAC have been demonstrated in various tissue fibroblasts, including HSCs (reviewed in [7779]). Early studies have documented that quiescent HSCs have high levels of pCREB, 
which decreases upon HSC activation and can be restored with activation of PKA [80-83]. Our previous studies demonstrated that primary HSCs do not express cAMP degrading PDE4 when they are quiescent; however, the expression of three PDE4 subfamilies of proteins, PDE4A, B, and D, increases upon the early stage of their activation [63]. Notably, culturing freshly isolated rat HSCs in the presence of a PDE4 specific inhibitor significantly attenuated the expression of HSC activation markers, aSMA, and Colla1, and prevented their phenotypic change into myofibroblasts [63]. These data strongly suggest that the induction of PDE4 and the consequent decrease in cAMP signaling are required for HSC activation. We also observed a persistent increase in hepatic PDE4 expression in a cholestatic liver injury rat model of fibrosis. We recently examined the expression of hepatic PDE4 in livers of severe AH patients with fibrosis and observed a significant upregulation of PDE4 expression (both mRNA and protein) in comparison to healthy donor livers [84]. Several publications have shown the beneficial effects of cAMP-specific PDE inhibitors in attenuating hepatic fibrosis in vivo [85-87].

cAMP/EPAC signaling as a regulator of fibrosis in different tissues is also well recognized [77, 78]. Mechanisms of EPAC mediated regulation of fibrosis include activation/differentiation of tissue-resident cells, epithelial-mesenchymal transformation (EMT), and recruitment of bone marrow progenitors [77, 78]. TGF $\beta 1$, the most potent profibrogenic cytokine, decreased EPAC1 expression in fibroblasts [78]. A critical mechanism of EPAC-mediated effects on fibroblast activation seems to be mediated by a small GTPase, Rho-A kinase (ROCK) [88]. Early studies in HSCs identified Rho-kinase as a regulator of actin cytoskeleton reorganization. This cytoskeleton reorganization leads to a phenotypic change of HSCs into myofibroblasts [89]. It has also been shown that 
fibrotic livers from both humans and rodents have decreased levels of EPAC, which correlate with increased levels of phospho-Myosin Light Chain (p-MLC), a downstream target of ROCK1 [88]. Later studies confirmed that Rho-kinase signaling regulates HSC activation and migration [90-92]. Several other studies demonstrated the beneficial effects of selective delivery of Rho-kinase inhibitor to HSCs on hepatic fibrosis development in vivo [93-95]. EPAC also plays a critical role in liver fibrosis. EPAC1 was decreased, while EPAC2 protein was elevated in activated rat HSCs [96]. Stimulation of the EPAC1/Rap1 pathway reduced the proliferation of HSCs, aSMA expression, and collagen type I and III syntheses [96]. Another study reported that EPAC-1 expression decreased in fibrotic livers compared to normal livers in the $\mathrm{CCl}_{4}$ mouse model and human fibrotic livers [88]. In the same study, administration of prostaglandin E2, a cAMP activator, attenuated plateletderived growth factor (PDGF)-induced proliferation and migration of stellate cells by the restoration of EPAC1 [88]. The same effect was shown using both EPAC and PKA agonists [88]. Notably, PDGF and transforming growth factor-beta (TGF $\beta 1$ ) suppressed EPAC1 mRNA expression levels in isolated HSCs, with no effect on PKA [88]. It was also shown in a study that although PKA did not reduce $\alpha$-SMA levels; however, it mediates phosphorylation of regulatory proteins required for mesenchymal transformation (EMT) [97]. EMT is one of the mechanisms leading to fibrosis. Cells of epithelial phenotype transition to a mesenchymal phenotype through increases in $\alpha$-SMA and decreases in Ecadherin expression. Both PKA and EPAC were shown to attenuate TGF- $\beta$-mediated reduction in E-cadherin expression [98]. 


\subsection{In vivo models of liver fibrosis}

Multiple in vivo murine fibrosis models made it easier to study liver fibrosis. The choice between these models is usually based on which cause of liver fibrosis is examined and the study aims/objectives. We will focus on two main categories of these in vivo models: hepatotoxin induced models and cholestatic models.

\subsubsection{Hepatotoxin induced liver fibrosis models:}

As reviewed in [99], The most commonly used hepatotoxin to induce liver fibrosis is carbon tetrachloride (CCl4). In mice, periodic intra peritoneal administration of CCl4 at a dose of $0.5-2 \mathrm{ml} / \mathrm{kg}$ body weight 2-3 times per week results in development of highly reproducible liver fibrosis within 4-6 weeks. Oral gavage is an alternative administration route however, it is associated with high rates of early mortality. CCl4 can be also administered by inhalation mainly for induction of liver cirrhosis and portal hypertension however, it needs appropriate equipment and operator training. Different mice strains show variable susceptibility to CCl4-induced liver fibrosis. BALB/c mice are the most susceptible and FVB/N mice are the least susceptible. C57Bl/6 mice are the most frequently used in $\mathrm{CCl}_{4}$-induced liver fibrosis models because of the availablility of respective knockouts. $\mathrm{C} 57 \mathrm{Bl} / 6$ mice show intermediate liver fibrosis in response to $\mathrm{CCl}_{4}$.

$\mathrm{CCl}_{4}$ is transformed by CYP2E1 to toxic trichloromethyl radical (CCl*3) [100]. This radical reacts with nucleic acids, proteins, and lipids, thereby impairing key cellular processes resulting in altered lipid metabolism (fatty degeneration and steatosis) and decreased protein synthesis. Oxygenation of $\mathrm{CCl}^{*} 3$ radical forms trichloromethylperoxy 
radicals ( $\mathrm{CCl} 3 \mathrm{OO} *)$ and this leads to aggravation of lipid peroxidation and the destruction of polyunsaturated fatty acids. Consequently, Global alteration of membrane permeability in all cellular compartments occur causing generalized hepatic damage characterized by inflammation, fibrosis, cirrhosis and HCC [100].

Liver fibrosis in response to $\mathrm{CCl}_{4}$ can be divided into acute injury, early fibrosis and advanced fibrosis phases. In acute injury phase, liver necrosis triggers inflammation and Kupffer cells activation resulting in secretion of cytokines, chemokines and other proinflammatory mediators [101]. This is followed by proliferation of hepatocytes and nonparenchymal cells to mediate regeneration at around 48 hours after the first CCl4 injection [101]. That's why acute single CCl4 injection can also be used as a model to investigate toxic hepatic injury. Significant fibrosis and scarring usually occurs after 2-3 weeks of $\mathrm{CCl}_{4}$ administration. This is associated with significant induction of profibrotic markers. Advanced bridging fibrosis develops usually after 4-6 weeks of CCl4 administration. Moreover, $\mathrm{CCl}_{4}$-induce fibrosis shows complete resolution within several weeks of discontinuation of $\mathrm{CCl}_{4}$ administration [101]. In conclusion, $\mathrm{CCl}_{4}$ model shows significant similarity with human liver fibrosis pathology including inflammation, regeneration, fibrosis development and regression.

Other toxicant induced liver fibrosis models include thioacetamide (TAA), Dimethyl nitrosamine (DMN) and Diethyl nitrosamine (DEN) models. TAA is bioactivated in the liver via CYP2E2 by oxidation producing toxic S-oxide and S-S dioxide radicals. TAA can be administered intraperitoneally at a dose of $150-200 \mathrm{mg} / \mathrm{kg}$ body weight 3 times a week [102]. This leads to significant centrilobular necrosis, elevated liver transaminases 
and fibrosis within 6 weeks [102]. DMN and DEN are much less frequently used in fibrosis research. Their mutagenic and carcinogenic properties cause overlapping and mutated signaling pathways leading to difficulty in assessment if profibrotic mechanisms in these models [102]. However, it has been described that intraperitoneal injection of 10 $\mathrm{mg} / \mathrm{kg}$ body weight of these compounds results in liver fibrosis within 4 weeks [102].

\subsubsection{Cholestatic models}

Cholestatic biliary epithelial damage is one of the major causes of liver fibrosis. Multiple causes lead to cholestatic inflammation and fibrosis including; autoimmune hepatitis, primary biliary cirrhosis and biliary tree obstruction. Animal models are a useful tool to study cholestatic liver fibrosis. The most common murine models for cholestasis are surgical bile duct ligation model, multidrug resistant gene knock out model and primary biliary cirrhosis models. All these models show several characteristics of liver injury such as obstruction-induced biliary epithelial damage, periductular inflammation/mononuclear cellular infiltration. Proper model is chosen by the investigator based on his study aims and objectives.

Surgical bile duct ligation model is the most commonly used cholestatic murine model. The animal is anaesthetized followed by ligation/dissection of common extra-hepatic bile duct. Jaundice and significant liver fibrosis establish in mice and rats within 28 days [103]. Multiple variations in the surgical technique exist based on special study aims such as re-anastomosis after bile duct ligation, partial bile duct ligation and microsurgical ligation [103]. This model can be used to study cholestatic liver injury in normal mice as well as transgenic mice. 
One of the most commonly used genetically modified mice in cholestatic liver fibrosis are the multidrug resistant (MDR) knock out mice. MDR2 in mice and MDR3 in humans are class III multi-drug-resistant P-glycoproteins which act as canalicular phospholipid translocators and are involved in biliary phospholipid (phosphatidylcholine) excretion [104]. Mdr2 gene knockout in mice leads to deficiency in phosphatidylcholine into the bile. This triggers inflammatory cholangitis, portal inflammation and ductular proliferation starting shortly after birth. The pathology progress to end stage liver fibrosis resembling sclerosing cholangitis and biliary fibrosis within 3-6 months [104].

In addition to the above-mentioned models, dietary models leading to cholestatic liver injury have been introduced. An example of these models is 3,5-diethoxycarbonyl-1,4dihydrocollidine (DDC) model. Feeding mice a diet supplemented with $0.1 \%$ DDC for 8 weeks leads to increased biliary porphyrin secretion [104]. This leads to ductular inflammatory reaction within one week. Expression of cytokines such as vascular cell adhesion molecule, osteopontin and TNF- $\alpha$ is upregulated in duct epithelial cells. Multiple pathological features are observed including pericholangitis, inflammatory mononuclear cellular infiltration and activation of periductal myofibroblasts, causing biliary liver fibrosis that resembles sclerosing cholangitis in humans [104].

\subsection{In vitro cell lines for liver fibrosis}

Cell lines are an important alternative to primary cells offering the advantages of unlimited supply and ease of use [139]. In this section, the most commonly used human and rodent hepatic stellate cell lines will be discussed. 


\subsubsection{Human HSC cell lines}

The most commonly used human HSC line is the Lieming Xu (LX)-2. which was created from LX-1 line. LX-1 cells were generated by transfection of hepatic stellate cells with the pRSVTag plasmid which encodes the SV40 large T antigen under the control of a rous sarcoma virus (RSV) promoter. LX-2 cells were established by selecting the LX-1 cells that were able to grow under reduced serum conditions (1\% FBS) [105]. Both cell lines express the key fibrotic receptors resembling primary HSCs, including platelet derived growth factor receptor $\beta$ ( $\beta$ PDGF-R), obese receptor long form (Ob-RL), and discoidin domain receptor 2 (DDR2). They also express proteins involved in matrix remodelling; matrix metalloproteinase (MMP), tissue inhibitor of matrix metalloproteinase (TIMP) [105]. Moreover, LX1 and LX2 cells were shown to express the key fibrotic proteins as well as HSC activation markers such as $\alpha$-SMA, procollagen and HSP47 in response to TGF $\beta 1$ stimulation. They also retain other key features of primary HSCs including the expression of intermediate filaments (Vimentin and Glial fibrillary acidic protein) and uptake/metabolism of retinoic acid [105]. The unique advantages of LX2 cells over LX1 are their viability in serum free media and high transfectability. That's why LX-2 cells are considered as a model of choice for investigating the signaling pathways in HSC activation because of their great similarity to in vivo HSC activation.

Another human HSC line is the human telomerase reverse transcriptase cells (hTERT cells). These cells were generated by Schnabl et al by isolation of human HSCs from the liver and its infection with a retrovirus expressing hTERT [106]. Functional expression of 
the telomerase catalytic subunit prevent telomere shortening with repeated cell division and DNA replication. This extend the life span of various normal human cells. hTERT cells did not show any oncogenic transformation and exhibited characteristics of activated HSCs by Microarray and RT-PCR [106]. Moreover, plating hTERT cells on a basement membrane-like matrix reverts them toward a more quiescent phenotype [106].

\subsection{Hypothesis and aims}

Previous study done by our group showed that PDE4 enzymes are upregulated in primary rat HSCs upon spontaneous activation in culture [63]. In this study, freshly isolated primary rat HSCs did not express PDE4 proteins, however, upon their attachment, they were rapidly induced. Intertestingly, during this process, cells did not express aSMA indicating that PDE4 induction preceded the process of HSC activation. Importantly, PDE4 inhibition by Rolipram attenuated primary rat HSC activation and expression of COL1A1 and aSMA gene expression [63]. Additionally, PDE4 inhibition significantly attenuated TGF $\beta 1$ mediated fibrogenic signaling in a rat bile duct ligation model ofcholestatic liver injury/fibrosis [63].

Preliminary studies done by our group (unpublished data) showed that PDE4 inhibition by Rolipram (targeted to the liver) attenuated $\mathrm{CCl}_{4}$-induced liver fibrosis in mice. Importantly, PDE4 inhibition by Rolipram significantly attenuated collagen deposition as demonstrated by Sirius red staining and hydroxyproline assay. We also observed significant attenuation of ECM remodeling enzymes, matrix metalloprotease 2 and tissue inhibitor of metalloprotease 2 in Rolipram treated group. Moreover, significant attenuation of heat 
shock protein 47 (chaperone protein involved in collagen synthesis) and lysyl oxidase enzymes (enzymes involved in collagen cross linking) occurred in Rolipram treated group.

These observations led us to hypothesize that PDE4 inhibition prevents development of liver fibrosis by attenuating TGFß1-induced activation of hepatic stellate cells. To test this hypothesis, we performed in vitro studies using LX2 hepatic stellate cell line. Additionally, we studied the effect of PDE4 inhibition on fibrogenic pathways in $\mathrm{CCl}_{4}$ liver fibrosis model by proteomic analysis. 


\section{CHAPTER 2}

\section{MATERIALS AND METHODS}

\section{Experimental Animals}

C57BL6/J mice were purchased from Jackson Laboratory (Bar Harbor, ME) and housed in a specific pathogen-free animal facility accredited by the Association for Assessment and Accreditation of Laboratory Animal Care under a protocol approved by the University of Louisville Institutional Animal Care and Use Committee (for Dr. Leila Gobejishvili). The room was maintained on a 12-hour light/dark cycle.

\section{Experimental Design}

Mice were subjected to $\mathrm{CCl}_{4}$ injection intraperitonealy at a dose of $1 \mathrm{mg} / \mathrm{kg}$ body weight twice a week for the duration of 4 weeks. One group of mice received Rolipram (PDE4 inhibitor, $3 \mathrm{mg} / \mathrm{kg}$ body weight) intraperitonealy twice a week, a day after $\mathrm{CCl}_{4}$ administration (Figure 6). 48 hours after the last dose of $\mathrm{CCl}_{4}$, mice were anesthetized, whole blood was collected from vena cava and liver tissue was harvested for protein and gene expression analysis. One peace of liver was fixed in $10 \%$ neutral buffered formalin for histological assessment. 


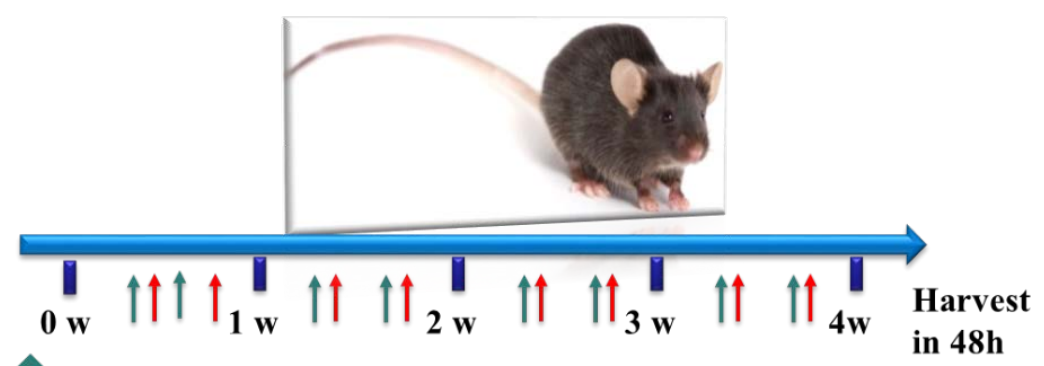

$\mathrm{CCl}_{4}, 1 \mathrm{mg} / \mathrm{kg}$ body weight, twice a week

Rolipram, $3 \mathrm{mg} / \mathrm{kg}$ body weight, twice a week, day after $\mathrm{CCl}_{4}$

Figure 6. Chronic $\mathrm{CCl}_{4}$ mouse model experimental approach

\section{Cell Culture}

LX2 human hepatic stellate cell line (LX2-HSCs) was obtained from SigmaAldrich. Cells were cultured in Dulbecco's modified Eagle's medium (DMEM) (ATCC, Manasas, VA) with $2 \%$ fetal bovine serum (FBS) and $1 \%$ penicillin/streptomycin at $37^{\circ} \mathrm{C}$ in $5 \% \mathrm{CO}_{2}$ and plated at density of 0.18 million/well in 6 well plates and 0.4 million in 100 mm dishes. Cells were starved in serum-free DMEM overnight and treated with human recombinant TGF $\beta 1(2.5 \mathrm{ng} / \mathrm{ml})$ with and without Rolipram $(10 \mu \mathrm{M})$. Cells were collected at early timepoints (30 minutes and 90 minutes) and late timepoint (24 hours) for RT qPCR and western blotting.

\section{Proteomic analysis}

Protein lysates preparation: (1) Protein lysates were prepared from liver tissue using an equal volume of $2 \%$ sodium dodecylsulfate (SDS) dissolved in $0.1 \mathrm{M}$ Tris- $\mathrm{HCl} \mathrm{pH} 8.5$ containing 1X HALTTM protease/phosphatase inhibitors (Thermo Fisher, Waltham, MA) supplemented with 0.5mM EDTA and concentrations estimated using a detergent compatible DC protein assay (BioRad, Inc Hercules, CA). Protein lysates $(100 \mu \mathrm{g})$ were 
trypsinized using the modified Filter-Aided Sample Preparation (FASP) method. (2) Protein samples were reduced with dithiothreitol (DTT), denatured with 8M urea and alkylated with iodoacetamide followed by centrifugation through a high molecular weight cutoff centrifugal filter (Millipore, 10k MWCO). After overnight digestion with sequencing grade Trypsin (Promega), the digested proteins were desalted and concentrated using an Oasis HLB 1cc (30mg) Extraction Cartridge (Waters Corporation, Milford, MA) using a modified protocol for extraction of the digested peptides. (3) Prior to peptide quantification by NanoDrop2000 A205 measurement. Protein digested samples $(50 \mu \mathrm{g})$ were labeled with TMT TMT10plex ${ }^{\mathrm{TM}}$ Isobaric Label Reagent Set (Thermo Fisher, Waltham, MA). To remove excess labeling reagent the samples were concentrated and desalted with Oasis HLB Extraction cartridges (Waters Corp, Milford, MA).

High pH reversed phase fractionation: Samples (90-100ug) were then subjected to high $\mathrm{pH}$ reversed phase separation at $37^{\circ} \mathrm{C}$ using a Dionex U3000SD uHPLC system (ThermoFisher Scientific, Waltham, MA, USA) with a BEH XBridge C18 $5 \mu \mathrm{m} 3.0 \mathrm{x}$ $150 \mathrm{~mm}$ column (Waters Corp, Milford, MA) for $70 \mathrm{~min}$ and at $300 \mu \mathrm{L} / \mathrm{min}$ flow rate with 5-88\% acetonitrile gradient buffered with $10 \mathrm{mM}$ ammonium formate pH10.0. Fractions were collected with an AFC-3000 fraction collector and after concatenation a total of 19 fractions were used for proteomic analysis to measure TMT-labeled peptides.

LC-MS/MS data collection and analysis: Briefly, the fractionated samples were analyzed by an Easy-nLC 1000 and Orbitrap Elite MS system (Thermo Scientific). Peptides in samples were trapped on an Acclaim PepMap 100 75 $\mu \mathrm{m}$ x 2cm, nanoViper

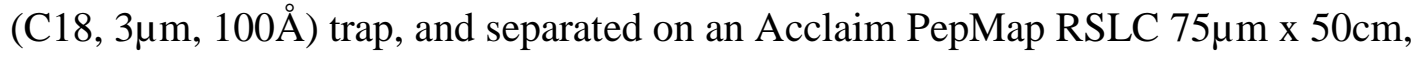
nanoViper (C18, 2 $\mu \mathrm{m}, 100 \AA$ ) column (ThermoFisher Scientific, Waltham, MA), both 
heated at $50^{\circ} \mathrm{C}$, with a 120 min binary solvent gradient ( $2 \%$ acetonitrile with $0.1 \%$ formic acid and $80 \%$ acetonitrile with $0.1 \%$ formic acid). Eluate from the column was directly ionized by a nanospray source and analyzed by the mass spectrometer in DDA mode. The mass resolution was set to 60,000 for MS and MS/MS. AGC was set to 5e5 for MS and 1e4 for MS/MS and the isolation window was set to $1 \mathrm{~m} / \mathrm{Z}$. Acquired data were processed by Proteome Discoverer (PD v1.4.1.14) with Sequest HT and Mascot (v.2.5.1) search engines and reviewed sequences from the reference mouse proteome from UniprotKB (downloaded on 7/18/2018). Data were searched considering two missed tryptic cleavages, static modifications of amino groups at $\mathrm{N}$-termini of all peptides and lysine residues by TMT 10plex tag, and cysteine residues by carbamidomethylation as well as dynamic modification of methionine oxidation. Match tolerances were set to $50 \mathrm{ppm}$ and 0.05 Da for precursor and fragment ions, respectively. The target-decoy PSM validator node in PD v1.4 was used to estimate the false discovery rates (FDR) for peptide identifications. The result files from Proteome Discoverer were loaded into Scaffold Q+S v4.4.5. Scaffold was used to calculate the false discovery rate using the Scaffold Local FDR and Protein Prophet algorithms. Peptides were accepted if the identification had probability greater than $99.9 \%$ and parent mass error within 2ppm. Proteins were accepted if they had a probability greater than $99.9 \%$ and at least one peptide. Proteins were grouped into clusters to satisfy the parsimony principle. For relative quantification, intensity of report ions from peptides specific to the protein group were used to calculate the relative abundance of identified proteins. Hepatic proteins that had significance abundance were imported into Clarivate analytics software MetaCore and pathway enrichment analysis was performed. 


\section{Scratch assay}

LX2 cells were plated at 0.2 million cells/well in 6 well plates in DMEM (ATCC, Manasas, VA) with 2\% FBS and 1\% penicillin/streptomycin at $37^{0} \mathrm{C}$ in $5 \% \mathrm{CO}_{2}$ for 24 hours. Then the medium was aspirated, and the cell-coated surface was scraped with a 1 ml pipette tip in a single stripe. The medium was changed to serum free DMEM and cells were treated with TGF $\beta 1(2.5 \mathrm{ng} / \mathrm{ml})$ and TGF $\beta 1+$ Rolipram $(10 \mu \mathrm{M})$. Plates were allowed to heal at $37{ }^{\circ} \mathrm{C}$ in $5 \% \mathrm{CO}_{2}$ for 24 hours inside the incubator. Migration of cells was observed with an inverted microscope and photographed at 0 hours and 24 hours. The average extent of wound closure was evaluated by multiple measurements of the width of the wound space.

\section{RT qPCR}

Total RNA was isolated from $50 \mathrm{mg}$ of liver tissue using TRIzol Reagent (Invitrogen, Carlsbad, CA) and cDNA was made using XLAscript cDNA master mix (BioExcell, Bristol, PA). Real time PCR was performed with an ABI prism 7500 sequence detection system and PerfeCTa SYBR Green FastMix, Low ROX reagents (Quanta Biosciences, Inc.). The relative gene expression was analyzed using $\Delta \Delta \mathrm{CT}$ method by normalizing to GAPDH gene expression in all experiments. Data are presented as fold change over the values for untreated control group. Primer sequences are listed in Table 1. 
Table 1. qPCR primer sequences.

\begin{tabular}{|c|c|c|}
\hline Target & Forward Primer (5' to $3^{\prime}$ ) & Reverse Primer (3' to 5') \\
\hline$h-E T-1$ & CAAGCAGGAAAAGAACTCAG & CTGGTTTGTCTTAGGTGTTC \\
\hline$h-G A P D H$ & CCATGGGGAAGGTGAAGGTC & GAAGGGGTCATTGATGGCAAC \\
\hline
\end{tabular}

\section{Western Blotting}

Mouse livers and LX2 hepatic stellate cell whole protein lysates were prepared by homogenization in radioimmunoprecipitation (RIPA) buffer mixed with Halt protease and phosphatase inhibitor cocktail (Thermo Fisher) followed by centrifugation at $14000 \mathrm{rpm}$ for 15 minutes at $4^{0} \mathrm{C}$ to remove insoluble material. For LX2 cells, cytoplasmic isolation was done using cytoplasmic lysis buffer (10 mM HEPES, $1.5 \mathrm{mM} \mathrm{MgCl}$, 10 mM KCl, 0.5 mM DTT, 1 mM EDTA, Ph 7.9) with 0.1\% NP40. Halt protease and phosphatase inhibitor cocktail (Thermo Fisher) was then added (1\%); Lysates were kept on ice for 1 hour followed by centrifugation at 4000rpm for 4 minutes at $4^{0} \mathrm{C}$ and cytoplasmic supernatant was isolated from nuclear pellet. Nuclear lysis buffer (20 mM HEPES, 1.5 mM MgCl 2,520 mM NaCl, 0.1 mM EDTA, 0.5 mM DTT, 25\% glycerol, Ph 7.8) with 0.2\% NP40 was used for lysis of nuclear pellet. Protein concentrations were determined by Bradford assay using reagents from Bio-Rad (Hercules, CA) with bovine serum albumin as a standard. $25 \mu \mathrm{g}$ of total protein in the 6X sample buffer was loaded onto a BioRad acrylamide gel (Biorad cat. no. 4561086) and separated at 100 volts for 80 minutes. Proteins were electro-blotted on a polyvinylidene difluoride membrane at a current not exceeding 300 milliamps for 1.5 hours. Membranes were blocked with 5\% milk in tris-buffered saline (TBST) for 1 hour and incubated overnight with a primary antibody at $4^{0} \mathrm{C}$. The membranes were washed with 
TBST and incubated with the suitable secondary antibodies, and imaged using a BioRad Chemidoc $^{\mathrm{TM}}$ imaging system.

\section{Statistical Analysis}

Data are shown as mean \pm standard deviation (SD). GraphPad Prism 8 software (GraphPad Software, San Diego, CA) was used to perform unpaired t-tests (for two-group comparison) or one-way ANOVA tests with post hoc analysis (for more than two groups). Data are considered significant at $\mathrm{p}<0.05\left({ }^{*} \mathrm{p}<0.05,{ }^{* *} \mathrm{p}<0.01\right.$ and $\left.{ }^{* * *} \mathrm{p}<0.001\right)$. 


\section{CHAPTER 3}

\section{RESULTS}

\section{The proteomic analysis shows that PDE4 inhibition has significant effects on liver fibrosis pathways}

Liver proteomic analysis was performed to examine the pathways affected by PDE4 inhibition. This analysis was performed by COBRE proteomic core with the help of Dr. Merchant. Metacore software was used for pathway analysis. As shown in Table 2, PDE4 inhibition had a significant effect on proteins involved in the development/perpetuation of liver fibrosis.

Affected proteins are part of either liver fibrosis signaling pathways e.g., WNT3A and IL1 $\beta$ or cytoskeleton remodeling and cell adhesion pathways e.g., ROCK/MLCP. These results suggest some pathways/target proteins through which PDE4 inhibition mediates its attenuation of initiation and perpetuation of liver fibrosis. 
Table 2. PDE4 inhibition significantly affects pathways related to liver fibrosis in the $\mathrm{CCl}_{4}$ mouse model.

\begin{tabular}{|c|c|c|}
\hline Liver fibrosis & Cytoskeleton remodeling & Cell adhesion \\
\hline $\begin{array}{c}\text { 1-ECM remodeling } \\
\text { DAB2 (Disabled 2) } \\
\text { COL1A1 } \\
\text { COL1A2 } \\
\text { Biglycan } \\
\text { 2-WNT3A signaling (Survival): } \\
\beta \text {-catenin } \\
\text { 3- TNF } \alpha \text { and IL-1 } \beta \text { signaling (recruitment of } \\
\text { inflammatory cells) } \\
\text { MYD88 } \\
\text { TRADD } \\
\text { RIPK1 } \\
\text { ICAM1 }\end{array}$ & $\begin{array}{c}\text { PRK1 } \\
\text { ROCK (1\&2) } \\
\text { MLCP (reg) } \\
\alpha \text {-adducin } \\
\text { MRLC } \\
\text { Myosin II complex (Myh4,6,9,10,11,12b) } \\
\text { MyHC protein group (actb) } \\
\text { Acto-myosin } \\
\text { MLCK (Mylk) } \\
\text { PAK (p21-activated kinase) } \\
\text { FilaminA } \\
\text { ERM protein group (Ezr, Msn, Rdx) } \\
\text { Contractin (Scr substrate) } \\
\text { Cofilin 1\&2 } \\
\text { Vinculin }\end{array}$ & $\begin{array}{c}\text { Col1a1 } \\
\text { Col1a2 } \\
\alpha 1 / \beta 1 \text { integrin } \\
\text { a5/ß1 integrin } \\
\text { FAK (focal adhesion kinase 1) (PMID 28642549) } \\
\text { GRB2 (growth factor receptor-bound protein 2) } \\
\text { Erk1, } 2 \\
\text { MYLK1 (Myosin light chain kinase 1) }\end{array}$ \\
\hline
\end{tabular}

\section{PDE4 inhibition attenuated HSC activation and decreased TGF $\beta$ canonical signaling protein pSMAD3 in LX2 HSCs}

LX2 human hepatic stellate cells were cultured as mentioned before. Cells were treated with TGF $\beta 1(2.5 \mathrm{ng} / \mathrm{ml})$ and a group of cells were pretreated with Rolipram $(10 \mu \mathrm{M})$ before TGF $\beta 1$. We collected the cells after 30 minutes and made cytoplasmic lysates to examine for pSMAD3 expression by western blot. To assess if PDE4 inhibition attenuates TGF $\beta 1$ induced $\alpha$ smooth muscle actin (aSMA), a critical HSC activation marker, we collected cells after 24 hours and performed Western blot. PDE4 inhibition by Rolipram decreased TGF $\beta 1$-induced pSMAD3 and aSMA in LX2 hepatic stellate cells (Fig.7A and 1B). These data show that PDE4 inhibition attenuates TGF $\beta$ 1-induced HSC activation. 


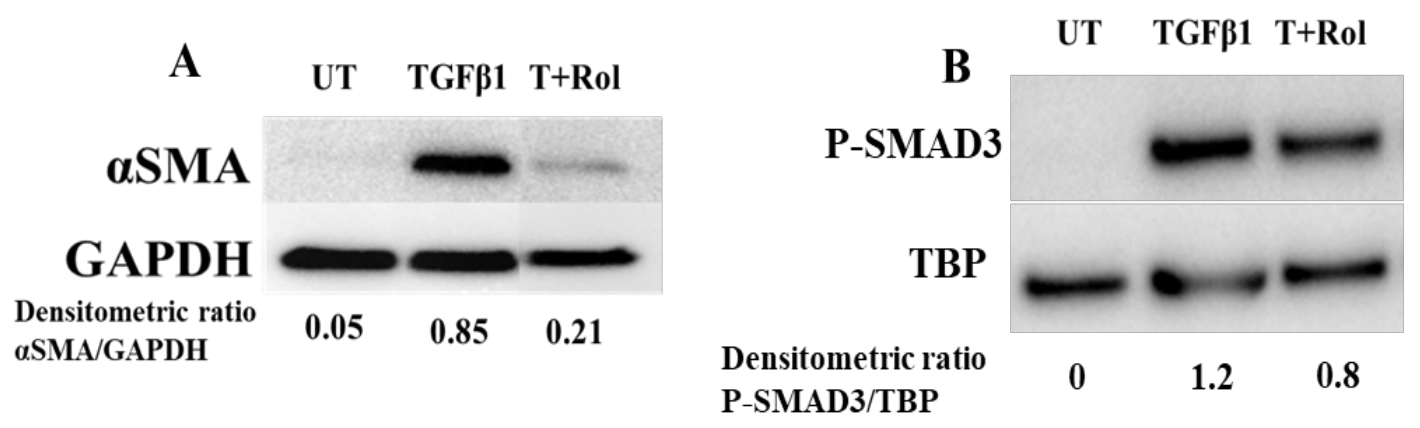

Figure 7. (A) PDE4 inhibition decreased $\alpha$ SMA expression in LX2 cells . (B) PDE4 inhibition attenuated TGF 1 induced pSMAD3 expression in LX2 HSCs. This indicates that PDE4 inhibition decrease liver fibrosis by attenuation of TGF $\beta 1$ canonical signaling pathway causing decreased HSC activation.

\section{PDE4 inhibition decreased expression of endothelin 1 and activation of myosin light chain}

Actomyosin-mediated contractility is one of the key mechanisms for the generation of mechanical stress and cell motility in HSCs. Actomyosin contractility is controlled by phosphorylation of myosin light chain, which is a regulated by myosin light chain kinase (MLCK) and myosin light chain phosphatase (MLCP) enzymes. Phosphorylated myosin light chain (pMLC) generates mechanical force on actin filaments mediating HSC motility/contractility.

Endothelin-1 (EDN1), released by HSCs in response to TGFß1 during fibrosis, induces HSC motility/contractility promoting the perpetuation of liver fibrosis.

We examined the effect of PDE4 inhibition on perpetuation of liver fibrosis by examining its effect on pMLC and EDN1 in TGF $\beta 1$-stimulated LX2 human HSC line.

LX2 HSCs were treated with TGF $\beta 1(2.5 \mathrm{ng} / \mathrm{ml})$ and a group of cells were pretreated with Rolipram $(10 \mu \mathrm{M})$ before TGF $\beta 1$. We collected the cells after 90 minutes and made 
cytoplasmic lysates to examine for pMLC expression by western blot. We also isolated RNA from cells treated with and without Rolipram and TGF $\beta 1,24$ hours after TGF $\beta 1$ treatment to examine for EDN-1 mRNA expression by RT qPCR.

TGF $\beta 1$ administration increased $E D N-1$ expression at mRNA level (Fig. 8A) and pMLC at the protein level (Fig. 8B). Notably, cells treated with TGF $\beta 1+$ Rol had significantly less pMLC and EDN-1 expression compared to TGF $\beta 1$ alone (Fig.8A, B).
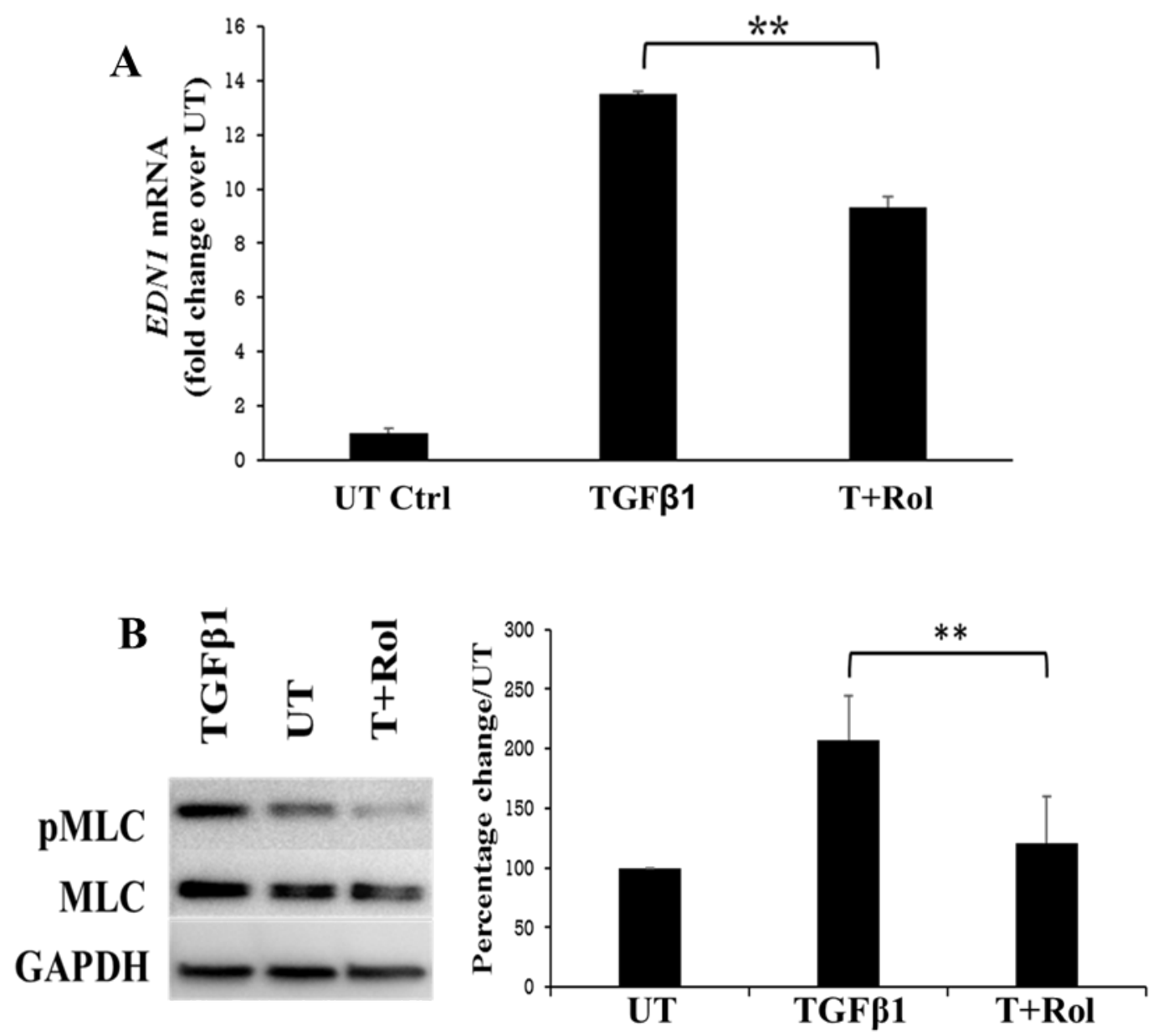

Figure 8. (A) PDE4 inhibition significantly decreased EDN-1 gene expression in LX2 cells . (B) PDE4 inhibition attenuated TGF $\beta 1$ induced pMLC expression in LX2 HSCs. Graph showing collected 
densitometry for multiple western blots normalized to untreated (UT) control. All data are represented as mean $\pm \mathrm{SD},{ }^{* *} \mathrm{p}<0.01$

\section{PDE4 inhibition decreases wound healing ability of LX2 HSCs}

We studied the wound healing and migration properties of LX2 HSCs using scratch assay. A scrape wound created on the LX2 cells treated with either TGF $\beta 1$ alone or TGF $\beta 1+$ Rol was observed after 0 hours and 24 hours of incubation in serum free medium. LX2 cells treated with TGF $\beta 1$ were able to close $80 \%$ of the wound size in 24 hours. Rolipram significantly attenuated TGF $\beta 1$-induced cell migration/wound closure in LX2 cells by almost half in 24 hours (Fig.9). Taken together, these results demonstrate that PDE4 inhibition attenuates HSC motility/contractility, one of the main mechanisms involved in the perpetuation of liver fibrosis. 

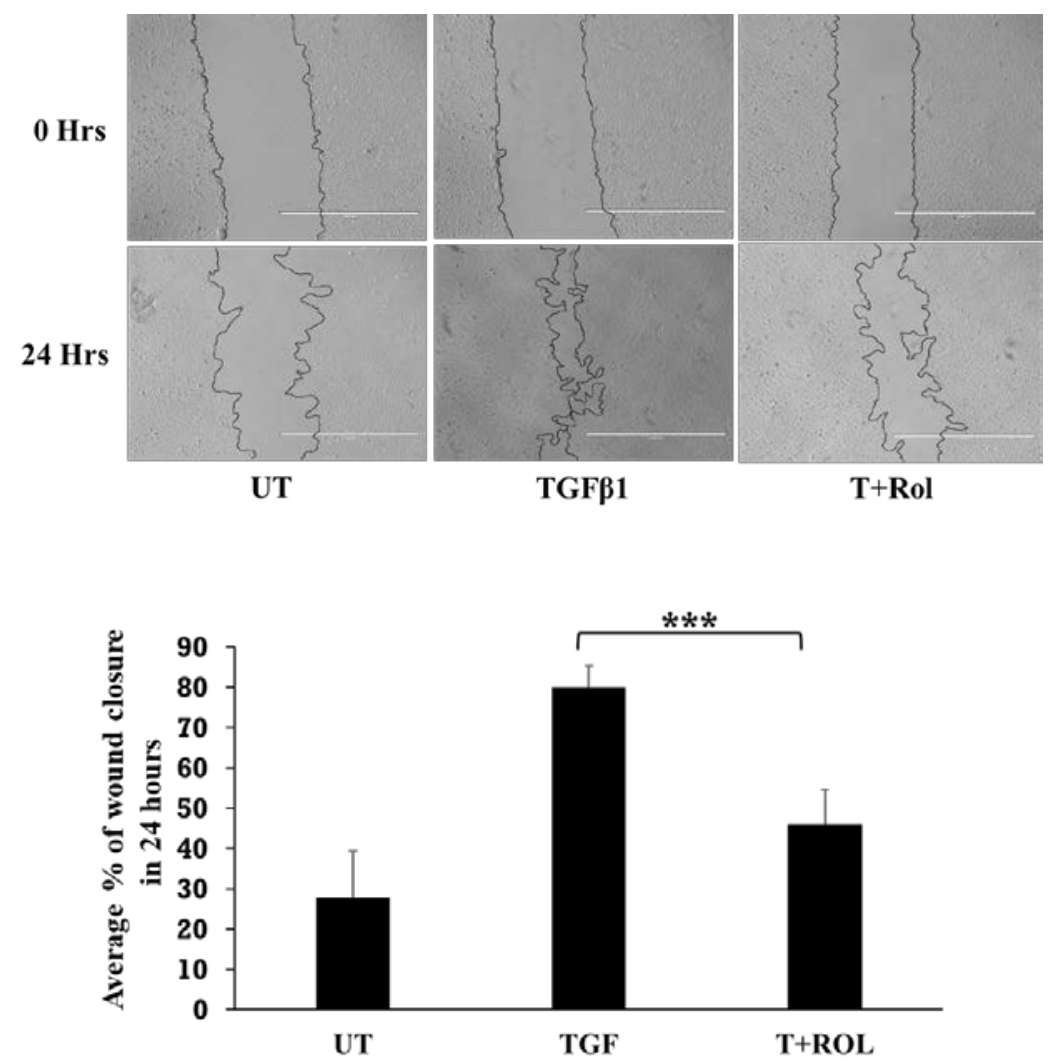

Figure 9. PDE4 inhibition significantly decreased TGF $\beta 1$-induced wound healing capacity in LX2 HSCs . Data in the graph are shown as the average percentage of wound closure in 24 hours. Data are presented as mean \pm SD, ${ }^{* * *} \mathrm{p}<0.001$. 


\section{CHAPTER 4}

\section{DISCUSSION}

PDE inhibition has been suggested to be an effective treatment strategy for several diseases, including liver diseases. PDE4 inhibition was shown to attenuate primary rat HSC activation markers in vitro. Importantly, previous studies done by our group showed that induction of PDE4 enzymes plays a pathogenic role the development of liver injury, inflammation and fibrosis in a rat model of cholestatic liver injury [63]. Therefore, we hypothesized that PDE4 inhibition inhibits TGF $\beta 1$ signalingin hepatic stellate cells (HSCs) and decreases their activation, and motility/contractility. In our experiments, PDE4 inhibition by Rolipram attenuated liver fibrosis, both in vivo and in vitro. Moreover, our group observed that there was a significant increase in hepatic PDE4 expression in alcoholic hepatitis human liver samples. These findings suggest PDE4 inhibition may be a novel drug target in liver fibrosis. That is extremely important since there are no FDAapproved therapies for liver fibrosis. Our findings also concur with previous reports that PDE4 inhibition effectively treats liver inflammation, ER stress, and ALD, which are common pathogenic mechanisms leading to liver fibrosis [84]. 
PDE4 inhibition decreases the rate of cAMP degradation leading to upregulation of cAMP signaling. It was shown in several studies that cAMP effector molecules (PKA and EPAC) have anti-fibrotic effects on fibroblasts (reviewed in [77-79]). Activated HSCs were shown to have lesser expression of pCREB (downstream cAMP effector) [80-83]. EPAC attenuates fibroblastic motility via its effect on Rho kinase (ROCK) which regulates actin polymerization [89]. Therfore, upregulation of cAMP signaling via PDE4 inhibition could be a potential strategy for treatment of liver fibrosis.

In our preliminary studies, $\mathrm{CCl}_{4}$ toxicant-induced liver fibrosis mice model was used. Interestingly, we observed that in the $\mathrm{CCl}_{4}$ mice model, PDE4 inhibition afforded excellent protection against liver fibrosis. Liver fibrosis is primarily driven by TGF $\beta 1$ induced HSCs activation, meaning that the beneficial effects of PDE4 inhibition in liver fibrosis may be mostly due to attenuation of TGF $\beta 1$ signaling/effects in HSCs.

We found significant effects of PDE4 inhibition on $\mathrm{CCl}_{4}$-induced liver fibrosis. Moreover, in our in vivo experiments, we did observe PDE4 inhibition attenuated TGF $\beta 1$ induced pSMAD3, a canonical signaling pathway molecule in the TGF 31 signaling pathway. Phospho-SMAD3 recruits other SMAD molecules. The SMAD complex then translocates to the nucleus and affects the expression of profibrotic genes. Interestingly, we obsreved a highly significant effect on collagen deposition and ECM remodeling enzymes in vivo, aSMA (a marker of HSC activation) and mediators of HSCs motility/contractility both in vitro and in vitro. In addition, proteomic analysis done on $\mathrm{CCl}_{4}$ mouse model showed a significant effect of PDE4 inhibition on pathways related to liver fibrosis, cell motility and adhesion. 
HSC motility is another aspect of liver fibrosis. It is mediated mainly by contractility and cytoskeleton remodeling. Once activated, HSCs undergo phenotypic change to contractile myofibroblasts, which resemble smooth muscle cells. Phosphorylation of myosin light chain (MLC) by myosin light chain kinase (MLCK) causes its activation. Activated MLC exerts tension by coupling to actin filaments leading to the generation of a contractile force. Focal adhesion proteins together with actin-myosin sliding mediate HSC motility/contractility. Endothelin-1 and TGF $\beta 1$ cause an increase in pMLC and HSCs contractility/motility [107]. PDE4 inhibition significantly decreased pMLC and ET-1 expression both in vivo in $\mathrm{CCl}_{4}$ treated mice and in vitro in LX2 HSCs.

Importantly, these results were strongly supported by a significant reduction in the wound healing ability of LX2 HSCs in the scratch assay. Moreover, endothelin-1 is a known vasoconstrictor and mediator of portal hypertension in the liver [108]. Thus, PDE4 inhibition may improve portal hypertension, a significant complication of liver fibrosis/cirrhosis.

Despite our results, more work has to be done to identify the molecular mechanisms by which downstream cAMP effectors (EPAC and PKA) attenuate TGF $\beta 1$ induced HSC activation. In addition, it's important to examine if PDE4 inhibition can be used as a therapeutic rather than preventive approach in liver fibrosis. That's what we are planning to do as explained later in our future directions. 


\section{CHAPTER 5}

\section{FUTURE DIRECTIONS}

In summary, Our work suggests that elevated cAMP via hepatic PDE4 inhibition attenuates liver fibrosis initiation and perpetuation. In vitro, PDE4 inhibition had a significant effect on TGF $\beta 1$ induced LX2 HSCs activation and motility/contractility by decreasing aSMA, pMLC and endothelin-1 expression. However, additional future work is necessary to validate PDE4 inhibition as a treatment strategy for liver fibrosis (Figure 12).

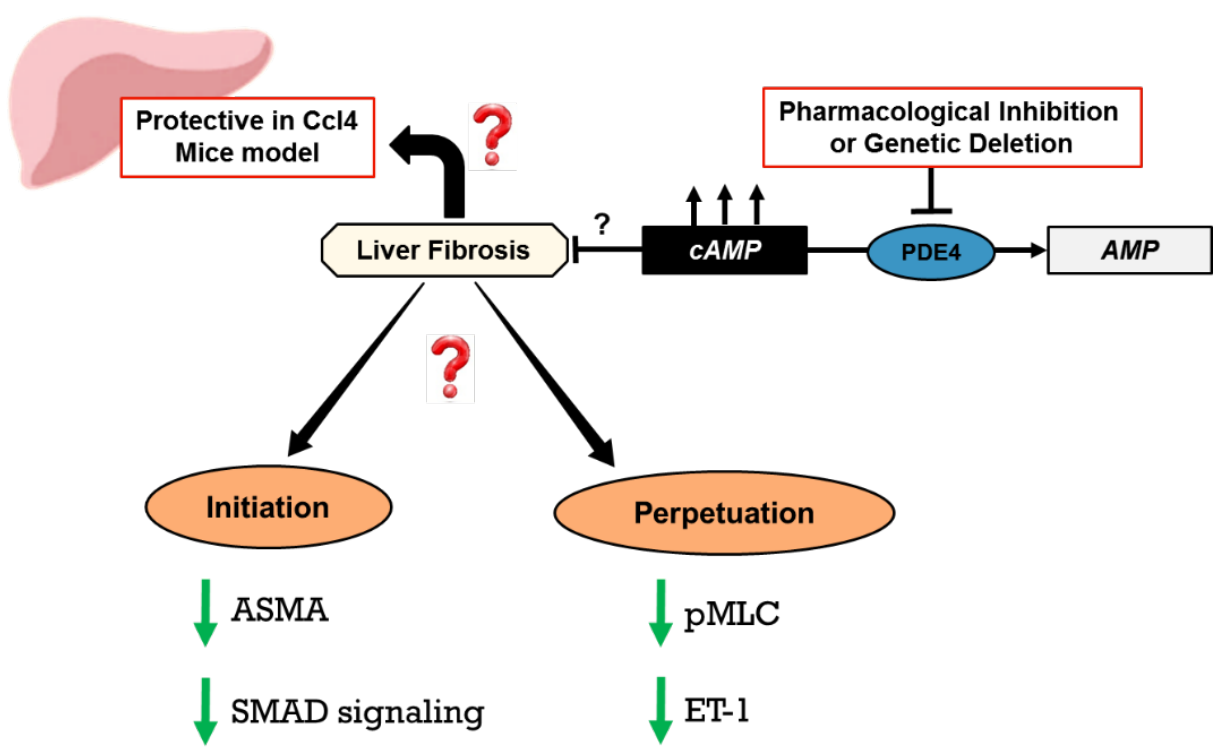

Figure 10. Summary figure. 
Two main questions will be answered through our future studies. First, which cAMP effector is involved in each of the effects we observed in our studies by PDE4 inhibition? Second, can PDE4 inhibition be used in treating rather than preventing liver fibrosis? In our future studies, we plan to investigate if PDE4 inhibition can treat $\mathrm{CCl}_{4}$ induced liver fibrosis rather than just prevent it. For making the studies more clinicallyrelevant, we plan also to use more clinically relevant PDE4 inhibitors (Roflumilast and D46) in our in vivo treatment studies.

In our future in vitro studies, we will elucidate the exact role of PKA and EPAC in mediating anti-fibrotic effects in hepatic stellate cells by using both pharmacological and siRNA approaches. Additionally, we are planning to determine the role of PDE4 subtypes in HSC activation/transdifferentiation and liver fibrosis development.

These future studies will clarify the mechanisms by which PDE4 inhibition and cAMP signaling attenuates liver fibrosis. It will also introduce a new potential therapy for liver fibrosis.

\section{Funding}

These studies were funded by the National Institute of General Medical Sciences of the National Institutes of Health under Award Number P20GM113226. 


\section{REFERENCES}

1. Corless, J.K. and H.M. Middleton, 3rd, Normal liver function. A basis for understanding hepatic disease. Arch Intern Med, 1983. 143(12): p. 2291-4.

2. Lerapetritou, M.G., et al., Tissue-level modeling of xenobiotic metabolism in liver: An emerging tool for enabling clinical translational research. Clin Transl Sci, 2009. 2(3): p. 228-37.

3. Geerts, A., History, heterogeneity, developmental biology, and functions of quiescent hepatic stellate cells. Semin Liver Dis, 2001. 21(3): p. 311-35.

4. Dixon, L.J., et al., Kupffer cells in the liver. Compr Physiol, 2013. 3(2): p. 785-97.

5. Lautt, W.W., Colloquium Series on Integrated Systems Physiology: From Molecule to Function to Disease, in Hepatic Circulation: Physiology and Pathophysiology. 2009, Morgan \& Claypool Life Sciences Copyright (C 2010 by Morgan \& Claypool Life Sciences.: San Rafael (CA).

6. Pellicoro, A., et al., Liver fibrosis and repair: immune regulation of wound healing in a solid organ. Nat Rev Immunol, 2014. 14(3): p. 181-94.

7. Kim, W.R., et al., Burden of liver disease in the United States: summary of a workshop. Hepatology, 2002. 36(1): p. 227-42.

8. Jefferies, M., et al., Update on global epidemiology of viral hepatitis and preventive strategies. World J Clin Cases, 2018. 6(13): p. 589-599.

9. Ward, J.W., The hidden epidemic of hepatitis C virus infection in the United States: occult transmission and burden of disease. Topics in antiviral medicine, 2013. 21(1): p. 15-19.

10. Perumpail, B.J., et al., Clinical epidemiology and disease burden of nonalcoholic fatty liver disease. World J Gastroenterol, 2017. 23(47): p. 8263-8276.

11. Arriazu, E., et al., Extracellular matrix and liver disease. Antioxid Redox Signal, 2014. 21(7): p. 1078-97.

12. Lo, R.C. and H. Kim, Histopathological evaluation of liver fibrosis and cirrhosis regression. Clin Mol Hepatol, 2017. 23(4): p. 302-307.

13. Pinter, M., et al., Cancer and liver cirrhosis: implications on prognosis and management. ESMO Open, 2016. 1(2): p. e000042.

14. Lu, P., et al., Extracellular matrix degradation and remodeling in development and disease. Cold Spring Harb Perspect Biol, 2011. 3(12).

15. Bedossa, P. and V. Paradis, Liver extracellular matrix in health and disease. J Pathol, 2003. 200(4): p. 504-15.

16. Ahmed, S.H., et al., Matrix metalloproteinases/tissue inhibitors of metalloproteinases: relationship between changes in proteolytic determinants of matrix composition and structural, functional, and clinical manifestations of hypertensive heart disease. Circulation, 2006. 113(17): p. 2089-96.

17. Xu, R., Z. Zhang, and F.S. Wang, Liver fibrosis: mechanisms of immune-mediated liver injury. Cell Mol Immunol, 2012. 9(4): p. 296-301.

18. Wells, R.G., Cellular sources of extracellular matrix in hepatic fibrosis. Clin Liver Dis, 2008. 12(4): p. 759-68, viii. 
19. Danen, E.H. Integrins: An Overview of Structural and Functional Aspects. 2013.

20. Bansal, R., et al., Integrin alpha 11 in the regulation of the myofibroblast phenotype: implications for fibrotic diseases. Exp Mol Med, 2017. 49(11): p. e396.

21. Honda, E., K. Yoshida, and H. Munakata, Transforming growth factor-beta upregulates the expression of integrin and related proteins in MRC-5 human myofibroblasts. Tohoku J Exp Med, 2010. 220(4): p. 319-27.

22. Zhou, W., et al., ADAMTS13 is expressed in hepatic stellate cells. Lab Invest, 2005. 85(6): p. 780-8.

23. Olaso, E., et al., DDR2 receptor promotes MMP-2-mediated proliferation and invasion by hepatic stellate cells. J Clin Invest, 2001. 108(9): p. 1369-78.

24. Seeff, L.B., Natural history of hepatitis C. Hepatology, 1997. 26(3 Suppl 1): p. 21s-28s.

25. Suk, K.T. and D.J. Kim, Staging of liver fibrosis or cirrhosis: The role of hepatic venous pressure gradient measurement. World J Hepatol, 2015. 7(3): p. 607-15.

26. Lee, U.E. and S.L. Friedman, Mechanisms of hepatic fibrogenesis. Best Pract Res Clin Gastroenterol, 2011. 25(2): p. 195-206.

27. Xu, J., et al., The types of hepatic myofibroblasts contributing to liver fibrosis of different etiologies. Front Pharmacol, 2014. 5: p. 167.

28. Uchio, K., et al., Cellular retinol-binding protein-1 expression and modulation during in vivo and in vitro myofibroblastic differentiation of rat hepatic stellate cells and portal fibroblasts. Lab Invest, 2002. 82(5): p. 619-28.

29. Dranoff, J.A. and R.G. Wells, Portal fibroblasts: Underappreciated mediators of biliary fibrosis. Hepatology, 2010. 51(4): p. 1438-44.

30. Lamireau, T., et al., Abnormal hepatic expression of fibrillin-1 in children with cholestasis. Am J Surg Pathol, 2002. 26(5): p. 637-46.

31. de Oliveira da Silva, B., L.F. Ramos, and K.C.M. Moraes, Molecular interplays in hepatic stellate cells: apoptosis, senescence, and phenotype reversion as cellular connections that modulate liver fibrosis. Cell Biol Int, 2017. 41(9): p. 946-959.

32. Cordero-Espinoza, L. and M. Huch, The balancing act of the liver: tissue regeneration versus fibrosis. J Clin Invest, 2018. 128(1): p. 85-96.

33. Fabregat, I., et al., TGF-B signalling and liver disease. Febs j, 2016. 283(12): p. 2219-32.

34. Zhao, X.-K., et al., Focal Adhesion Kinase Regulates Hepatic Stellate Cell Activation and Liver Fibrosis. Scientific Reports, 2017. 7(1): p. 4032.

35. Hanafusa, H., et al., Involvement of the $p 38$ mitogen-activated protein kinase pathway in transforming growth factor-beta-induced gene expression. J Biol Chem, 1999. 274(38): p. 27161-7.

36. Engel, M.E., et al., Interdependent SMAD and JNK signaling in transforming growth factor-beta-mediated transcription. J Biol Chem, 1999. 274(52): p. 37413-20.

37. Fan, X., et al., Attenuation of CCl4-induced hepatic fibrosis in mice by vaccinating against TGF-81. PLoS One, 2013. 8(12): p. e82190.

38. Ling, H., et al., Transforming growth factor $B$ neutralization ameliorates pre-existing hepatic fibrosis and reduces cholangiocarcinoma in thioacetamide-treated rats. PLoS One, 2013. 8(1): p. e54499.

39. Wong, L., et al., Induction of beta-platelet-derived growth factor receptor in rat hepatic lipocytes during cellular activation in vivo and in culture. J Clin Invest, 1994. 94(4): p. 1563-9.

40. Pinzani, M., PDGF and signal transduction in hepatic stellate cells. Front Biosci, 2002. 7: p. d1720-6. 
41. Pinzani, M., et al., Expression of platelet-derived growth factor in a model of acute liver injury. Hepatology, 1994. 19(3): p. 701-7.

42. Kocabayoglu, P., et al., 6-PDGF receptor expressed by hepatic stellate cells regulates fibrosis in murine liver injury, but not carcinogenesis. J Hepatol, 2015. 63(1): p. 141-7.

43. Borkham-Kamphorst, E., et al., Platelet-derived growth factor isoform expression in carbon tetrachloride-induced chronic liver injury. Lab Invest, 2008. 88(10): p. 1090-100.

44. Shao, R., W. Yan, and D.C. Rockey, Regulation of endothelin-1 synthesis by endothelinconverting enzyme-1 during wound healing. J Biol Chem, 1999. 274(5): p. 3228-34.

45. Reynaert, H., et al., Hepatic stellate cells: role in microcirculation and pathophysiology of portal hypertension. Gut, 2002. 50(4): p. 571-81.

46. Guo, C.Y., et al., Effects of endothelin-1 on hepatic stellate cell proliferation, collagen synthesis and secretion, intracellular free calcium concentration. World J Gastroenterol, 2004. 10(18): p. 2697-700.

47. Huang, G. and D.R. Brigstock, Regulation of hepatic stellate cells by connective tissue growth factor. Front Biosci (Landmark Ed), 2012. 17: p. 2495-507.

48. Abdelmegeed, M.A., et al., CYP2E1 potentiates binge alcohol-induced gut leakiness, steatohepatitis, and apoptosis. Free Radic Biol Med, 2013. 65: p. 1238-1245.

49. Benyon, R.C. and M.J. Arthur, Extracellular matrix degradation and the role of hepatic stellate cells. Semin Liver Dis, 2001. 21(3): p. 373-84.

50. Iredale, J.P., Hepatic stellate cell behavior during resolution of liver injury. Semin Liver Dis, 2001. 21(3): p. 427-36.

51. Yang, L., et al., Vascular endothelial growth factor promotes fibrosis resolution and repair in mice. Gastroenterology, 2014. 146(5): p. 1339-50.e1.

52. Kantari-Mimoun, C., et al., Resolution of liver fibrosis requires myeloid cell-driven sinusoidal angiogenesis. Hepatology, 2015. 61(6): p. 2042-55.

53. Elnagdy, M., et al., CAMP Signaling in Pathobiology of Alcohol Associated Liver Disease. Biomolecules, 2020. 10(10).

54. Sutherland, E.W. and T.W. Rall, Fractionation and characterization of a cyclic adenine ribonucleotide formed by tissue particles. J Biol Chem, 1958. 232(2): p. 1077-91.

55. Stanley McKnight, G., Cyclic AMP second messenger systems. Current Opinion in Cell Biology, 1991. 3(2): p. 213-217.

56. Steegborn, C., Structure, mechanism, and regulation of soluble adenylyl cyclases similarities and differences to transmembrane adenylyl cyclases. Biochim Biophys Acta, 2014. 1842(12 Pt B): p. 2535-47.

57. Pierce, K.L., R.T. Premont, and R.J. Lefkowitz, Seven-transmembrane receptors. Nat Rev Mol Cell Biol, 2002. 3(9): p. 639-50.

58. Taylor, S.S., et al., Structural framework for the protein kinase family. Annu Rev Cell Biol, 1992. 8: p. 429-62.

59. Taskén, K., et al., Structure, function, and regulation of human cAMP-dependent protein kinases. Adv Second Messenger Phosphoprotein Res, 1997. 31: p. 191-204.

60. Sassone-Corsi, P., Transcription factors responsive to cAMP. Annu Rev Cell Dev Biol, 1995. 11: p. 355-77.

61. Robichaux, W.G., 3rd and X. Cheng, Intracellular CAMP Sensor EPAC: Physiology, Pathophysiology, and Therapeutics Development. Physiol Rev, 2018. 98(2): p. 919-1053.

62. Conti, M. and J. Beavo, Biochemistry and physiology of cyclic nucleotide phosphodiesterases: essential components in cyclic nucleotide signaling. Annu Rev Biochem, 2007. 76: p. 481-511. 
63. Gobejishvili, L., et al., Rolipram attenuates bile duct ligation-induced liver injury in rats: a potential pathogenic role of PDE4. J Pharmacol Exp Ther, 2013. 347(1): p. 80-90.

64. Baillie, G.S., G.S. Tejeda, and M.P. Kelly, Therapeutic targeting of 3',5'-cyclic nucleotide phosphodiesterases: inhibition and beyond. Nat Rev Drug Discov, 2019. 18(10): p. 770796.

65. Lugnier, C., et al., Cyclic nucleotide phosphodiesterases: New targets in the metabolic syndrome? Pharmacol Ther, 2020. 208: p. 107475.

66. Ahmad, F., et al., Cyclic nucleotide phosphodiesterase $3 B$ is a downstream target of protein kinase $B$ and may be involved in regulation of effects of protein kinase $B$ on thymidine incorporation in FDCP2 cells. J Immunol, 2000. 164(9): p. 4678-88.

67. Johnstone, T.B., et al., CAMP Signaling Compartmentation: Adenylyl Cyclases as Anchors of Dynamic Signaling Complexes. Mol Pharmacol, 2018. 93(4): p. 270-276.

68. Dessauer, C.W., Adenylyl cyclase--A-kinase anchoring protein complexes: the next dimension in CAMP signaling. Mol Pharmacol, 2009. 76(5): p. 935-41.

69. Hayes, J.S., L.L. Brunton, and S.E. Mayer, Selective activation of particulate CAMPdependent protein kinase by isoproterenol and prostaglandin E1. J Biol Chem, 1980. 255(11): p. 5113-9.

70. Stangherlin, A., et al., Analysis of compartmentalized CAMP: a method to compare signals from differently targeted FRET reporters. Methods Mol Biol, 2014. 1071: p. 5971.

71. Berrera, M., et al., A toolkit for real-time detection of CAMP: insights into compartmentalized signaling. Handb Exp Pharmacol, 2008(186): p. 285-98.

72. Zaccolo, M., et al., Heterogeneity of second messenger levels in living cells. Novartis Found Symp, 2001. 239: p. 85-93; discussion 93-5, 150-9.

73. Ariga, M., et al., Nonredundant function of phosphodiesterases $4 D$ and $4 B$ in neutrophil recruitment to the site of inflammation. J Immunol, 2004. 173(12): p. 7531-8.

74. Jin, S.L. and M. Conti, Induction of the cyclic nucleotide phosphodiesterase PDE4B is essential for LPS-activated TNF-alpha responses. Proc Natl Acad Sci U S A, 2002. 99(11): p. 7628-33.

75. Jin, S.L., et al., Specific role of phosphodiesterase 4B in lipopolysaccharide-induced signaling in mouse macrophages. J Immunol, 2005. 175(3): p. 1523-31.

76. Méhats, C., et al., PDE4D plays a critical role in the control of airway smooth muscle contraction. Faseb j, 2003. 17(13): p. 1831-41.

77. Insel, P.A., et al., CAMP and Epac in the regulation of tissue fibrosis. Br J Pharmacol, 2012. 166(2): p. 447-56.

78. Yokoyama, U., et al., The cyclic AMP effector Epac integrates pro- and anti-fibrotic signals. Proc Natl Acad Sci U S A, 2008. 105(17): p. 6386-91.

79. Lopez-Sanchez, I., et al., GIV/Girdin is a central hub for profibrogenic signalling networks during liver fibrosis. Nat Commun, 2014. 5: p. 4451.

80. Houglum, K., K.S. Lee, and M. Chojkier, Proliferation of hepatic stellate cells is inhibited by phosphorylation of CREB on serine 133. J Clin Invest, 1997. 99(6): p. 1322-8.

81. Kawada, N., et al., Inhibition of myofibroblastic transformation of cultured rat hepatic stellate cells by methylxanthines and dibutyryl cAMP. Dig Dis Sci, 1996. 41(5): p. 1022-9.

82. Li, G., Q. Jiang, and K. Xu, CREB family: A significant role in liver fibrosis. Biochimie, 2019. 163: p. 94-100.

83. Delaunay, M., et al., The Role of Cyclic AMP Signaling in Cardiac Fibrosis. Cells, 2019. 9(1). 
84. Rodriguez, W.E., et al., Phosphodiesterase 4 Inhibition as a Therapeutic Target for Alcoholic Liver Disease: From Bedside to Bench. Hepatology, 2019. 70(6): p. 1958-1971.

85. El Awdan, S.A., et al., Regression of fibrosis by cilostazol in a rat model of thioacetamideinduced liver fibrosis: Up regulation of hepatic CAMP, and modulation of inflammatory, oxidative stress and apoptotic biomarkers. PLoS One, 2019. 14(5): p. e0216301.

86. Abdel Kawy, H.S., Cilostazol attenuates cholestatic liver injury and its complications in common bile duct ligated rats. Eur J Pharmacol, 2015. 752: p. 8-17.

87. Han, K., Y. Zhang, and Z. Yang, Cilostazol protects rats against alcohol-induced hepatic fibrosis via suppression of TGF-B1/CTGF activation and the CAMP/Epac1 pathway. Exp Ther Med, 2019. 17(3): p. 2381-2388.

88. Schippers, M., et al., Upregulation of Epac-1 in Hepatic Stellate Cells by Prostaglandin E(2) in Liver Fibrosis Is Associated with Reduced Fibrogenesis. J Pharmacol Exp Ther, 2017. 363(2): p. 126-135.

89. Iwamoto, H., et al., A p160ROCK-specific inhibitor, Y-27632, attenuates rat hepatic stellate cell growth. J Hepatol, 2000. 32(5): p. 762-70.

90. van Beuge, M.M., et al., Reduction of fibrogenesis by selective delivery of a Rho kinase inhibitor to hepatic stellate cells in mice. J Pharmacol Exp Ther, 2011. 337(3): p. 628-35.

91. Hu, Y., et al., Hypoxia-inducible factor $1 \alpha$ and ROCK1 regulate proliferation and collagen synthesis in hepatic stellate cells under hypoxia. Mol Med Rep, 2018. 18(4): p. 39974003.

92. Görtzen, J., et al., Interplay of Matrix Stiffness and c-SRC in Hepatic Fibrosis. Front Physiol, 2015. 6: p. 359.

93. Zhou, W., et al., Inhibition of Rho-Kinase Downregulates Th17 Cells and Ameliorates Hepatic Fibrosis by Schistosoma japonicum Infection. Cells, 2019. 8(10).

94. van Dijk, F., et al., The antifibrotic potential of a sustained release formulation of a PDGFB-receptor targeted rho kinase inhibitor. J Control Release, 2019. 296: p. 250-257.

95. Okimoto, S., et al., Vitamin A-coupled liposomal Rho-kinase inhibitor ameliorates liver fibrosis without systemic adverse effects. Hepatol Res, 2019. 49(6): p. 663-675.

96. Yang, Y., et al., EPAC activation inhibits acetaldehyde-induced activation and proliferation of hepatic stellate cell via Rap1. Can J Physiol Pharmacol, 2016. 94(5): p. 498-507.

97. Pattabiraman, D.R., et al., Activation of PKA leads to mesenchymal-to-epithelial transition and loss of tumor-initiating ability. Science, 2016. 351(6277): p. aad3680.

98. Cheng, X., et al., Epac and PKA: a tale of two intracellular CAMP receptors. Acta Biochim Biophys Sin (Shanghai), 2008. 40(7): p. 651-62.

99. Scholten, D., et al., The carbon tetrachloride model in mice. Lab Anim, 2015. 49(1 Suppl): p. 4-11.

100. Weber, L.W., M. Boll, and A. Stampfl, Hepatotoxicity and mechanism of action of haloalkanes: carbon tetrachloride as a toxicological model. Crit Rev Toxicol, 2003. 33(2): p. 105-36.

101. Yanguas, S.C., et al., Experimental models of liver fibrosis. Arch Toxicol, 2016. 90(5): p. 1025-1048.

102. Delire, B., P. Stärkel, and I. Leclercq, Animal Models for Fibrotic Liver Diseases: What We Have, What We Need, and What Is under Development. J Clin Transl Hepatol, 2015. 3(1): p. 53-66.

103. Tag, C.G., et al., Bile duct ligation in mice: induction of inflammatory liver injury and fibrosis by obstructive cholestasis. J Vis Exp, 2015(96). 
104. Liedtke, C., et al., Experimental liver fibrosis research: update on animal models, legal issues and translational aspects. Fibrogenesis \& Tissue Repair, 2013. 6(1): p. 19.

105. Xu, L., et al., Human hepatic stellate cell lines, $L X-1$ and $L X-2$ : new tools for analysis of hepatic fibrosis. Gut, 2005. 54(1): p. 142-51.

106. Schnabl, B., et al., Immortal Activated Human Hepatic Stellate Cells Generated by Ectopic Telomerase Expression. Laboratory Investigation, 2002. 82(3): p. 323-333.

107. Rockey, D.C. and R.A. Weisiger, Endothelin induced contractility of stellate cells from normal and cirrhotic rat liver: implications for regulation of portal pressure and resistance. Hepatology, 1996. 24(1): p. 233-40.

108. Angus, P.W., Role of endothelin in systemic and portal resistance in cirrhosis. Gut, 2006. 55(9): p. 1230-2. 


\section{CURRICULUM VITAE}

Mohamed Elnagdy

5207 Rosette blvd, Louisville, KY, 40218 | 502-295-6150 |

mxamin01@louisville.edu

\section{Education}

University of Louisville

2018 - Present

Department of Pharmacology and Toxicology

MS/PhD Student

Cumulative GPA 3.7/4.0

Cairo University, Cairo, Egypt

2015

Medical Degree

Excellent with honor

GPA: $3.8 / 4.0$

Badr Language school, Cairo, Egypt

2007

Science major

Percent score: $\mathbf{9 9 . 8 \%}$

Matched in faculty of medicine Cairo university

\section{Experience}

1- Stanford health care, San Diego. CA, United States of America 07/2020-08/2020

Medical observer, Dr.Jonathan H. Lin

Observership in pathology in which I had the chance to meet regularly with

Dr.Lin and review gross specimens, microscopic slides, flow cytometry, and other relevant histo/clinical pathology for his assigned cases

2- Cairo University, Cairo, Egypt

04/2016-04/2018

Teaching assistant, Medical pharmacology department

I was involved in teaching medical pharmacology to undergraduate medical and dental students. I was also involved in clinical pharmacology research through the departmental clinical pharmacology unit. 
3- Abbasia Psychiatric hospital, Cairo, Egypt

04/2015-04/2016

Training graduate physician

I was involved in direct patient care and management under the supervision of residents and attending physicians.

4- JFK medical center, Edison, NJ, United States of America

Training medical student

Medical elective rotation during my obligatory internship year in neurology. I had the chance to attend the morning resident's meeting and observe/contribute to patient care and examination. I was also involved in case presentations.

5- Case Western Reserve University, Cleveland, Ohio, United States of America 04/2014-05/2014

Training medical student

Medical elective rotation during my obligatory internship year in psychiatry. I had the chance to attend the morning resident's meeting and observe/contribute to patient care and examination. I was also involved in case presentations.

\section{Awards and Honors}

1- KC Huang scholarship at University of Louisville

2- Elective courses: Certificate of Health Professional Education (University of Louisville)

3- Education Commission of Foreign Medical Graduates certificate, Philadelphia, USA

4- Author of 4 books explaining basic science for USMLE step 1

\section{Teaching experience}

1- Basic science lecturer for USMLE STEP 1 (Microbiology, Physiology, Pharmacology, Biochemistry, Pathology, Anatomy, Behavioral Science, Genetics, immunology), TA7 USMLE Preparation system, Smart vision training institute Cairo, Egypt

2- Clinical knowledge lecturer for USMLE STEP 2 CK (Internal medicine, Pediatrics, Obstetrics, Gynecology, Surgery), TA7 USMLE Preparation system, Smart vision training institute, Cairo, Egypt

3- Clinical Skills lecturer for USMLE STEP 2 CS, TA7 USMLE Preparation system, Smart vision training institute, Cairo, Egypt

4- Lecturer in medical pharmacology department, faculty of medicine, Cairo University

- Clinical pharmacology: Drugs in CVS disease 
- Clinical pharmacology: Drugs in the treatment of peptic ulcer

- Clinical Pharmacology: Drugs in Bronchial asthma

- Clinical Pharmacology: Drugs in special groups

5- Introduction to autonomic nervous system pharmacology, Dental school, University of Louisville, KY

6- Cholinergic agonists and Muscarinic antagonists, Dental school, University of Louisville, Louisville, KY

7- Nicotinic receptor drugs, Dental school, University of Louisville, Louisville, KY

8- Antiepileptic drugs, Dental school, University of Louisville, Louisville, KY

9- Drugs in CNS disorders, Dental hygiene, University of Louisville, Louisville, KY

10-Student clinical case presentations, Dental school, University of Louisville, Louisville, KY

\section{Publications}

Elnagdy, M.; Barve, S.; McClain, C.; Gobejishvili, L. cAMP Signaling in Pathobiology of Alcohol Associated Liver Disease. Biomolecules 2020, 10, 1433.

\section{Abstracts}

1- Mohamed Elnagdy, Walter Rodriguez, Yali Wang, Michael Merchant, Philip Bauer, Claudio Maldonado, Shirish Barve, Craig J. McClain, Leila Gobejishvili. DECREASED RHO KINASE SIGNALING AND CONTRACTILITY OF HEPATIC STELLATE CELLS BY PDE4 INHIBITOR ATTENUATES FIBROTIC PATHWAYS IN VIVO AND IN VITRO. AASLD abstracts. 2020, May; VOLUME 158(ISSUE 6): S-1384. Pub Status: Published.

2- Mohamed Elnagdy, Walter Rodriguez-Alvarez, Yali Wang, Michael L. Merchant, Claudio Maldonado, Shirish Barve, Craig McClain, Leila Gobejishvili. Attenuation of fibrogenesis by PDE4 inhibitor via decreased cytoskeleton remodeling, activation, and contractility of hepatic stellate cells. The journal of the federation of American societies for experimental biology. 2020, Apr; Volume 34(Issue S1): 1-1. Pub Status: Published.

3- Richa Singhal, Walter Rodriguez Alvarez, Yali Wang, Mohamed Elnagdy, Julia Chariker, Samuel W French Sr., Hidekazu Tsukamoto, Philip Bauer, Jingwen Zhang, Craig J. McClain, Shirish Barve, Claudio Maldonado, Leila Gobejishvili. RNA Sequencing Identifies Antifibrotic Pathways Affected By Phosphodiesterase 4 (PDE4) Inhibition In A Mouse Model Of Alcoholic Steatohepatitis (ASH). Hepatology journal of the American association for the study of liver diseases. 2019, Oct; Volume 72(Issue 1): 849A. Pub Status: Published. 